\title{
Cumulative staircase considerations for dynamic lotsizing when backlogging is allowed
}

\author{
Robert Grubbström
}

\section{Linköping University Post Print}

\section{Tweet}

N.B.: When citing this work, cite the original article.

Original Publication:

Robert Grubbström, Cumulative staircase considerations for dynamic lotsizing when backlogging is allowed, 2013, International Journal of Production Economics.

http://dx.doi.org/10.1016/j.ijpe.2013.05.027

Copyright: Elsevier

http://www.elsevier.com/

Postprint available at: Linköping University Electronic Press

http://urn.kb.se/resolve?urn=urn:nbn:se:liu:diva-96595 


\title{
Cumulative Staircase Considerations for Dynamic Lotsizing when Backlogging is Allowed
}

\author{
Robert W. Grubbström FVR RI, FLO K \\ Linköping Institute of Technology, SE-581 83 Linköping, Sweden \\ Mediterranean Institute for Advanced Studies, Primorski tehnoloski park, SI-5290 Šempeter pri Gorici, Slovenia \\ robert@grubbstrom.com
}

\begin{abstract}
The dynamic lotsizing problem concerns the determination of optimal batch quantities, when given required amounts appear at discrete points in time. The standard formulation assumes that no shortages are allowed and that replenishments are made instantaneously.
\end{abstract}

For the case when no shortage is allowed, previously it has been demonstrated that the innercorner condition for an optimal production plan in continuous time reduces the number of possible replenishment times to a finite set of given points at which either a replenishment is made, or not. The problem is thus turned into choosing from a set of zero/one decisions with $2^{n-1}$ alternatives, of which at least one solution must be optimal, where $n$ is the number of requirement events. Recently, the instantaneous replenishment assumption has been replaced by allowing for a finite production rate, which turned the inner-corner condition into a condition of tangency between the cumulative demand staircase and cumulative production.

In this paper we investigate relationships between optimal cumulative production and cumulative demand, when backlogging is permitted. The production rate is assumed constant and cumulative production will then be a set of consecutive ramps. Cumulative demand is a given staircase function. The Net Present Value (NPV) principle is applied, assuming a fixed setup cost for each ramp, a unit production cost for each item produced and a unit revenue for each item sold at the time it is delivered.

Among other results, it is shown that optimal cumulative production necessarily intersects the demand staircase. Instead of having $2^{n-1}$ production staircases as candidates for optimality, there are $2^{n-1}$ production structures as candidates. These are made up of sequences of batches, of which the set of batches may be optimised individually.

Results for the Average Cost approach are obtained from a zeroth/first order approximation of the objective function (NPV).

Keywords: Dynamic lotsizing, finite production rate, backlog, shortages, net present value, binary approach.

\section{Introduction}

The dynamic lotsizing problem is an extension of the classical EOQ lotsizing problem (Harris 1913). This extension was published by Harvey M. Wagner and Thomson M. Whitin in 1958 (Wagner and Whitin 1958). Requirements (demand) to be satisfied are distributed over time in given amounts, and the problem is to decide how much to produce at different times in order to optimise an objective function, in the simplest case minimising the total sum of holding costs and setup costs. 
Originally, production was assumed to take place instantaneously, but Roger Hill relaxed this assumption in 1997 (Hill 1997), allowing for a finite production rate.

The timing framework for the problem is usually a discrete-time scale, but it has been advocated by the current author that a continuous-time scale is a superior approach, including discrete time as a special case.

The objective function has traditionally involved the average cost (AC) approach. Against this stands the more sophisticated Net Present Value (NPV) principle, applying an interest rate for modelling capital costs, and the AC objective is normally obtained, when the NPV is approximated as a first/zeroth-order expansion in this interest rate. Applying NPV to lotsizing problems was probably first published by George Hadley in 1964 (Hadley 1964), but since then there have been several contributions, and the interest for using this principle appears to be increasing (Trippi and Levin 1974, Grubbström 1980, Kim, Philippatos and Chung 1986, Teunter and van der Laan 2002, Beullens and Janssens 2011).

The inner-corner condition is a necessary condition for optimality, when formulating requirements and production as cumulative functions of time. When production takes place instantaneously, both of these functions become staircase functions, and this necessary condition means that the optimal production staircase fits into the given demand staircase at inner corners as is illustrated in Figure 1. This condition is valid either the AC or the NPV principle is followed. It is also valid for a general assembly system (Grubbström, Bogataj and Bogataj 2009, Grubbström and Tang 2012).

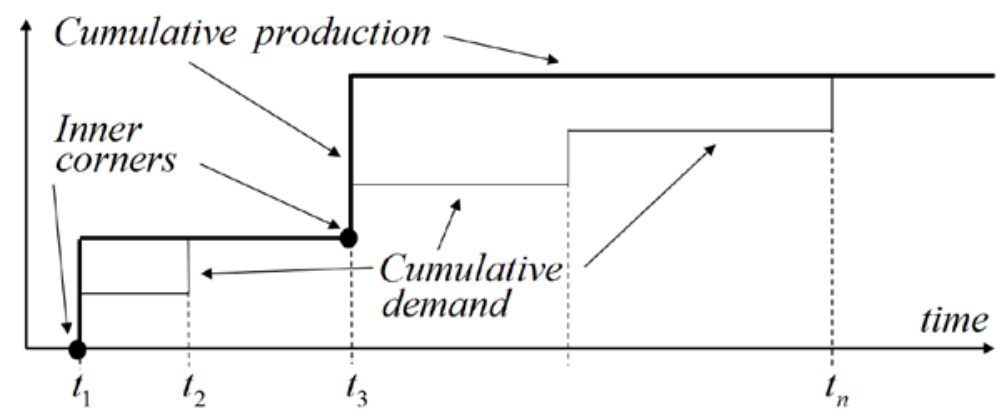

Figure 1. The inner-corner condition as a necessary provision for optimality.

The inner-corner condition limits the set of possible optimal solutions to a finite set of production staircases, since at each inner corner there is either a contact, or no contact. If there are $n$ steps in the demand staircase, the number of possible solutions become $2^{n-1}$, observing that there is always a contact at the very first inner corner. The dynamic lotsizing problem is then turned into a binary problem, at each demand event either production takes place or there is no production. This binary property was early recognised by Arthur Veinott Jr (Veinott 1969), although neither a continuous time scale, nor the NPV objective appear to have been applied to the problem formulation at that time.

As shown in a recent paper (Grubbström 2012), when the production rate is assumed to be finite, rather than that production takes place instantaneously, then inner-corners no longer appear. Instead a condition of tangency applies, and cumulative production now built up of a set of ramps either touch an upper corner of the demand staircase, or not, see Figure 2. The binary property thus is still valid, but it is now based on a different geometrical structure. 


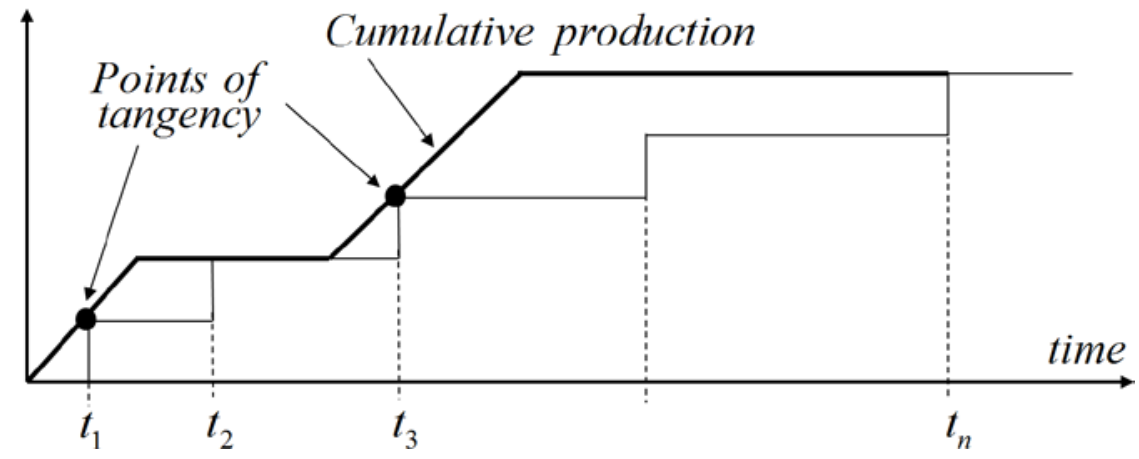

Figure 2. The "tangency" condition as a necessary prerequisite for optimality when no shortages are allowed.

Hitherto, the problem formulation has involved the assumption that all demand must be satisfied and that therefore no shortages are allowed. This assumption was relaxed by Y. Song and G.H. Chan in an article allowing for backlogs to be possible (Song and Chan 2005). The timing framework was there assumed to be discrete, and cumulative demand and production functions had not been introduced.

The problem treated in this paper is a follow-up of (Grubbström 2012) allowing for shortages to be possible at the cost of delaying the in-payments for backlogged items. Other penalties for negative consequences from shortages (such as badwill) are not considered here. Neither is the possibility of lost sales taken into consideration.

Cumulative functions are considered and economic consequences concern sales revenues and costs for setups and production. Basically an NPV approach is followed, and the corresponding AC results are compared with its first/zeroth order approximations. It is shown that the "inner-corner/tangency" condition now must be replaced by a weaker binary condition. Still there are $2^{n-1}$ possible structures to be chosen from, but these are not immediately recognised as to the timing of the individual production steps/ramps. Instead, each part of the cumulative production function involves an individual local optimisation.

A production structure eligible for optimality is thus a set of consecutive production ramps beginning and ending at points in time defined by the given requirements (the demand events) and covering all steps in the cumulative demand staircase. Each batch belonging to a structure may be optimised on its own, irrespective of which structure that currently is considered. Since there are $2^{n-1}$ structures, and only $n(n+1) / 2$ different batches, in general, a certain batch will belong to more than one structure. From a computational point of view, each batch can therefore be optimised on its own (by a suitably choice of its timing), and the dynamic lotsizing problem will then concern the question of matching the sequence of optimised batches in order to maximise the objective function in the NPV case, or minimise total costs in the AC case. The number of possible different batch sizes is seen from one opportunity to form a batch covering all $n$ steps (All-At-Once), two opportunities to form a batch covering $(n-1)$ steps, $\ldots$, and $n$ opportunities to form a batch covering just one step (Lot-For-Lot), together accounting for the arithmetic sum $\sum_{i=1}^{n} i=n(n+1) / 2$. It might be interesting to note that for very small values of $n$, the number of batch sizes is larger than the number of structures, i.e. $2^{n-1}<n(n+1) / 2$, for $2 \leq n \leq 4$. 
In Section 2 we first show that the segments of the optimal production structure start and end on levels belonging to the horizontal steps of the demand staircase. This is followed by a subsection treating conditions for optimal production segments (ramps, batches). It is shown that each batch has a unique optimal start time and that the optimal ramp necessarily intersects the demand staircase, when backlogging is allowed. Optimising the batches individually, provides a pool of, in total, $n(n+1) / 2$ batches to be chosen from when selecting the production structure maximising the overall NPV. Section 3 includes a numerical example illustrating our findings. This is followed by a short concluding section summarising our results.

\section{Introducing the possibility of shortages}

Table 1 lists the main notation to be used in the following. Additional notation is introduced when the need appears.

\begin{tabular}{|l|l|}
\hline$n$ & Given number of requirement events. \\
\hline$t_{i}$ & Given time at which the ith requirement appears, $i=1,2, \ldots, n$. \\
\hline$D_{i}$ & Given size of requirement at time $t_{i}, i=1,2, \ldots, n$. \\
\hline $\bar{D}_{i}=\sum_{j=1}^{i} D_{j}$ & Cumulative requirements immediately after time $t_{i} \cdot$ \\
\hline$p$ & Unit sales price (in-payment). \\
\hline$c$ & Unit production cost (out-payment). \\
\hline$K$ & Setup (ordering) cost for producing one batch (out-payment). \\
\hline$h$ & Inventory holding cost per unit and time unit. \\
\hline$b$ & Backlog cost per unit and time unit. \\
\hline$\rho$ & Continuous interest rate, per time unit. \\
\hline$Q$ & Batch size. \\
\hline$q$ & Finite production rate, units per time unit. \\
\hline$\beta$ & $\begin{array}{l}\text { Parameter indicating if setup costs are allocated to the beginning of } \\
\text { production intervals ( } \beta=0 \text { ) or to interval ends ( } \beta=1 \text { ). }\end{array}$ \\
\hline
\end{tabular}

\section{Table 1. Main notation.}

\subsection{Necessary condition for optimal production structure}

Our starting point consists of two geometrical structures, on the one hand the given cumulative demand (requirements) staircase, on the other the cumulative production function made up of a set of ramps, each having a slope of $q$, and joined by horizontal steps. Figure 3 illustrates the two functions. 


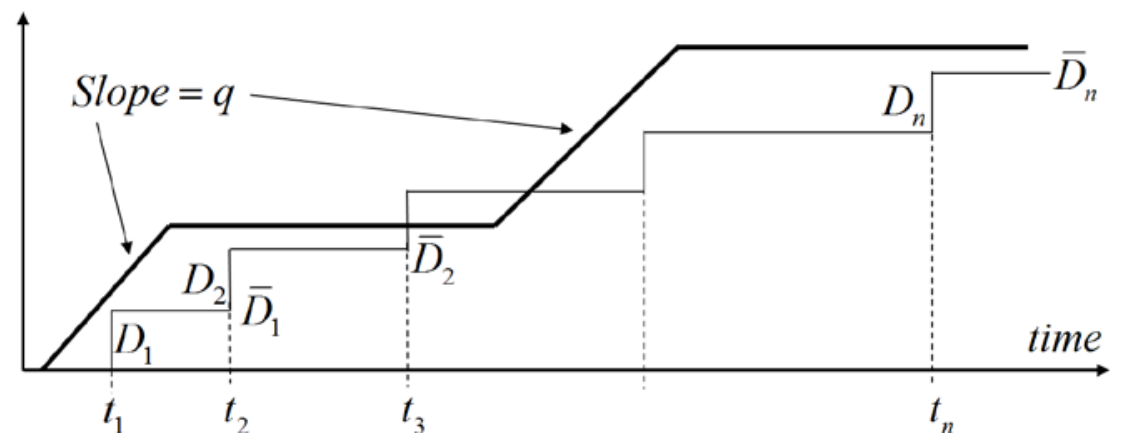

Figure 3. Cumulative demand staircase and the cumulative production function made up of a sequence of ramps.

If no shortages are allowed, then cumulative production must be at least cumulative demand, the case treated in (Grubbström 2012). However, we now allow for shortages to appear which means that the two curves may intersect each other. When cumulative production is above cumulative demand, the vertical distance in between represents items held in inventory, and in the opposite case the difference represents the current shortage of items. The vertical height of a ramp represents the batch size $Q$ of this production. The sequence of times $t_{i}, i=1,2, \ldots, n$, are defined from the demand staircase as the points when a requirement (demand) amounting to $D_{i}$ appears, $i=1,2, \ldots, n$, which are the vertical heights of the steps. Cumulative demand, immediately after $t_{i}$ is written $\bar{D}_{i}=\sum_{j=0}^{i} D_{j}$.

The objective function, when applying the NPV principle, is the sum of discounted inpayments less the sum of discounted out-payments. Out-payments are of two kinds, a unit production cost $c$ for each item produced and a fixed setup cost $K$ allocated either to the beginning of a production interval $(\beta=0)$ or to its end $(\beta=1)$. In-payments come when delivering items to customers with a sales price $p$, and they either appear at a demand event (if there are items available in inventory), or later when an item has been produced which can satisfy currently back-logged demand. The decision variables are the times at which each production ramp starts and the batch size of this ramp (alternatively the time at which the ramp ends).

Economic consequences for a batch thus come from three sources, namely setup costs, production costs and revenues. Setup consequences assume a given out-payment $K$ for each ramp; if it is located to some point in time $t=t^{\prime}$, then the contribution to NPV of this setup is $-K e^{-\rho t^{\prime}}$. As in (Grubbström 2012), we provide the two possibilities that $t^{\prime}$ is either at the beginning or at the end of the production interval. The production rate $q$ is assumed to be a given constant, and the production out-payment flow is assumed to be constant $c q$ during the production interval, giving an NPV contribution amounting to $-c q\left(1-e^{-\rho Q / q}\right) / \rho$ from a ramp of height $Q$, if payments are discounted to the beginning of this ramp.

Thirdly, we have the revenues contributing to NPV. If there is no shortage, then demand will contribute by $p D_{i} e^{-\rho t_{i}}$ (discounted to time $t=0$ ) for each such demand event. When there instead are shortages, they are all backlogged and this portion of demand is later satisfied as soon as production enables this. The NPV as a function of the start time $t$ of a given ramp (a batch) is given in Eq. (4) below. 
Our first conclusion concerns the fact that a production batch must start and end at a horizontal step of the cumulative demand staircase for the NPV to be maximised. A requirement for this to hold is that the unit sales price covers the unit production cost, i.e. $p>c$. We formulate this in the following theorem:

\section{Theorem 1}

On condition that $p>c$ and that all shortages are backlogged, it is always of advantage to start and end a production ramp from a horizontal step of the cumulative demand staircase. This has the consequence that the optimal production structure must be chosen from one of $2^{n-1}$ possible structures, where $n$ is the number of demand events.

\section{Proof}

Consider Figure 4.

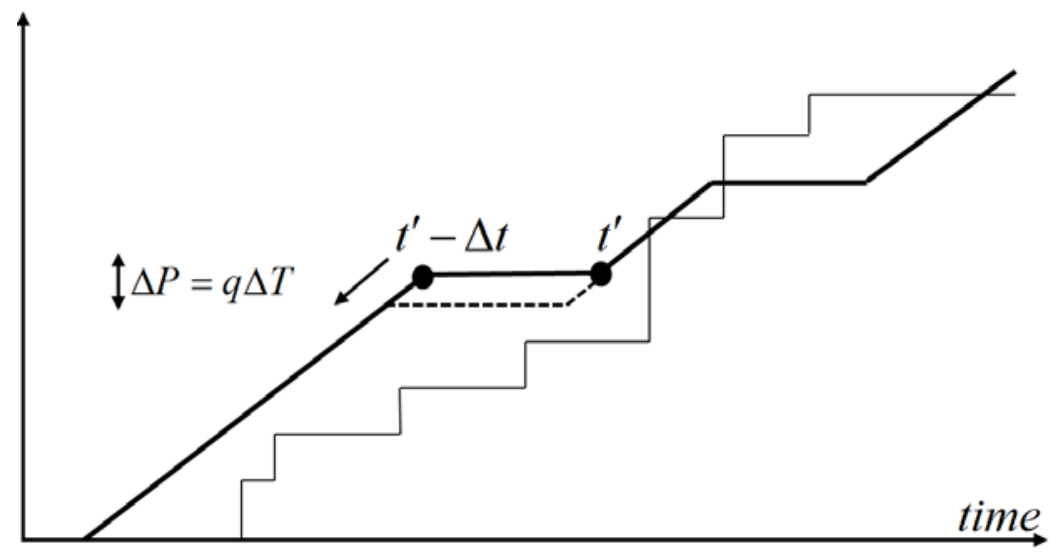

Figure 4. Moving production from one ramp to the next later.

Assume that a ramp begins above a horizontal demand step (at $t^{\prime}$ in Figure 4). The width of the horizontal production step is $\Delta t$. If the start of the ramp is advanced by $\Delta T=\Delta P / q$ (i.e. $\Delta P$ of production is moved from the left to the right ramp), then the change in NPV (discounted to $t=0$ ) is

$$
\Delta \mathrm{NPV}=\left(e^{\rho \Delta T}-1\right) e^{-\rho t^{\prime}}\left((c q / \rho)\left(e^{\rho \Delta t}-1\right)-K e^{\rho \beta \Delta t}\right),
$$

where $\beta=0$, if setups are allocated to the beginning of ramps, and unity if they are allocated to the ends.

If $\triangle \mathrm{NPV}$ is positive, it pays to move down, which is possible until the closest horizontal demand step below is reached, or if no such step is reached until the next lower horizontal production step. In the latter case, the left ramp disappears and the setup cost for this batch is also avoided.

If instead $\triangle \mathrm{NPV}$ is non-positive, it is possible to move up without any loss, involving a move of production from the right ramp to the left. This can be done until a vertical wall of the demand staircase is hit, or if no such wall is hit, until the next horizontal step of cumulative production is reached. If a vertical wall is passed (say at $t_{i}$ ), moving production to the left ramp means backlogs will decrease and sales from inventory will be enabled, see Figure 5. 


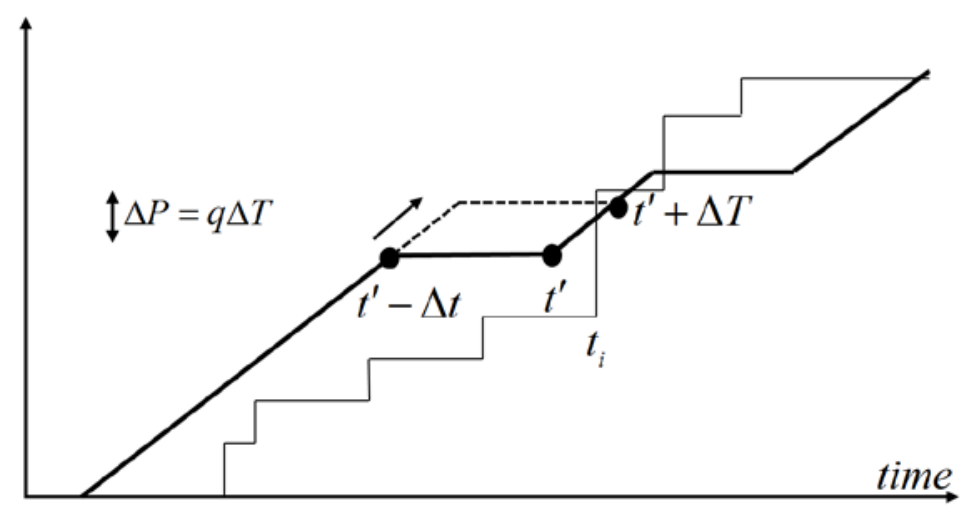

Figure 5. Moving production from a ramp passing a wall of the demand staircase to the next earlier ramp.

After passing a wall of the demand staircase, the NPV change from revenues becomes

$$
\begin{gathered}
\Delta \mathrm{NPV}_{\text {revenues }}=p q\left(t^{\prime}+\Delta T-t_{i}\right) e^{-\rho t_{i}}-p q\left(1-e^{-\rho\left(t^{\prime}+\Delta T-t_{i}\right)}\right) e^{-\rho t_{i}} / \rho= \\
=\left(p q e^{-\rho t_{i}} / \rho\right)\left(\rho\left(t^{\prime}+\Delta T-t_{i}\right)-\left(1-e^{-\rho\left(t^{\prime}+\Delta T-t_{i}\right)}\right)\right)>0,
\end{gathered}
$$

since $z-\left(1-e^{-z}\right)>0$, for any $z>0$.

So this increases the change in NPV from the move still further. Therefore the move should be made at least until the next horizontal step of cumulative production is reached. When this happens, the right ramp has disappeared, and its corresponding setup cost has been eliminated, which is a further motive for such a move.

Hence it can never pay to start production above a horizontal step of the demand staircase.

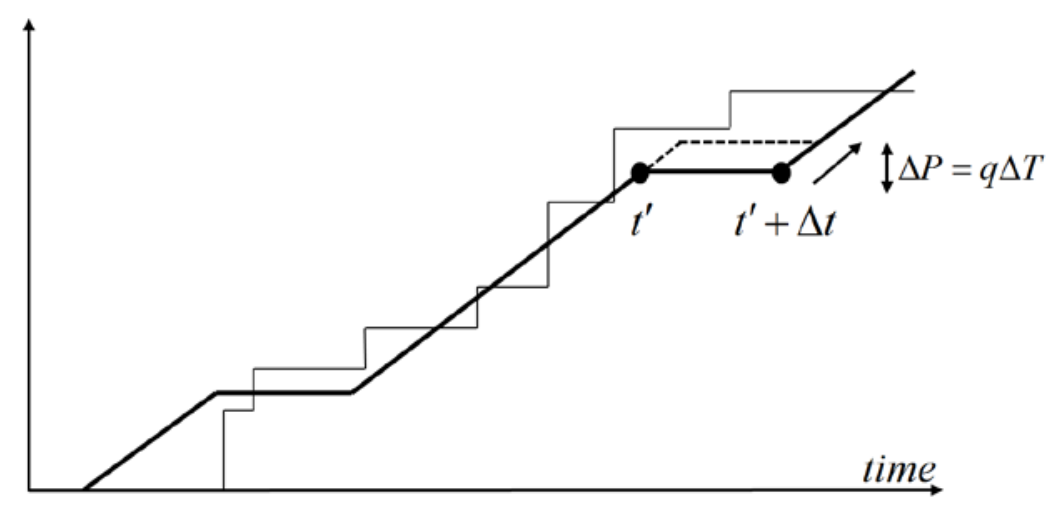

Figure 6. Moving production from a later to the next earlier ramp, when there are backlogs.

Assume now that a ramp ends at $t^{\prime}$, which is at a level below a horizontal step of cumulative demand, as depicted in Figure 6. If an increase in production by $\Delta P$ is made, prolonging production time by $\Delta T=\Delta P / q$ (and a similar decrease of the next batch), then the economic consequences are the following. The NPV of setup costs changes by $\Delta \mathrm{NPV}_{\text {setups }}=K e^{-\rho\left(t^{\prime}+(1-\beta) \Delta t\right)}\left(1-e^{-\rho \Delta T}\right)>0$, i.e. a decrease in cost consequences, and the NPV of 
production changes by $\Delta \mathrm{NPV}_{\text {production }}=-\frac{c q}{\rho}\left(1-e^{-\rho \Delta T}\right) e^{-\rho t^{\prime}}+\frac{c q}{\rho}\left(1-e^{-\rho \Delta T}\right) e^{-\rho\left(t^{\prime}+\Delta t\right)}$ $=-c q e^{-\rho t^{\prime}}\left(1-e^{-\rho \Delta T}\right)\left(1-e^{-\rho \Delta t}\right) / \rho$, the first an advantage and the second a disadvantage.

However, shortages also diminish, which increases the NPV of backlogged revenues by $\Delta \mathrm{NPV}_{\text {revenues }}=p q e^{-\rho t^{\prime}}\left(1-e^{-\rho \Delta T}\right)\left(1-e^{-\rho \Delta t}\right) / \rho$.

The total consequence of an upward move thus becomes

$$
\Delta \mathrm{NPV}=e^{-\rho t^{\prime}}\left(1-e^{-\rho \Delta T}\right)\left(K e^{-\rho(1-\beta) \Delta t}+(p-c) q\left(1-e^{-\rho \Delta t}\right) / \rho\right)>0,
$$

which is positive, considering that the unit production cost is covered by price, $p>c$. Therefore only an upward move is profitable.

The consequence of this reasoning is that it is necessary for each production ramp to start and end on a level given by the horizontal portions of the demand staircase in order for it to qualify as a candidate for optimality. This means that there will be $2^{n-1}$ structures available, either a ramp starts on a horizontal step, or it does not. The first ramp always starts from scratch, and it would never pay to produce more than what is totally demanded.

If we instead apply an AC approach, we need not consider setup cost consequences, since this approach does not take the timing of setups into consideration. The downward move in Figure 5 is always profitable, since a reduction in time-weighted holding costs amounts to $h$ times the area covered by the move, $h q \Delta T \Delta t$, and the upward move in Figure 6 is always

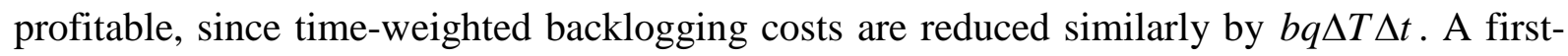
order expansion of $e^{\rho t^{\prime}} \Delta \mathrm{NPV}$ in (1) gives $e^{\rho t^{\prime}} \Delta \mathrm{NPV} \approx((c q / \rho)(\rho \Delta T)(\rho \Delta t)-(\rho \beta \Delta T) K)$ $\approx \rho c q \Delta T \Delta t>0$, neglecting interest consequences of setup costs, which shows the standard interpretation of the unit holding cost $h=\rho c$. A similar first-order expansion of $e^{\rho t^{\prime}} \Delta \mathrm{NPV}$ in (3) provides $e^{\rho t^{\prime}} \Delta \mathrm{NPV} \approx \rho(p-c) q \Delta T \Delta t$, which interprets the unit backlog cost parameter as $b=\rho(p-c)$.

\subsection{Optimisation for a given structure}

For each structure among the $2^{n-1}$ candidates for optimality, cumulative production will be a sequence of batches (ramps) starting at one level of the demand staircase and ending at a second level. Although the batch sizes are given, when having chosen one of these structures, their timing is still undetermined. We therefore attempt to find the optimal timing of each individual batch, confining our attention to a single batch of size $Q$ beginning at the cumulative demand level $\underline{D}$ and ending at the level $\underline{D}+Q$. 


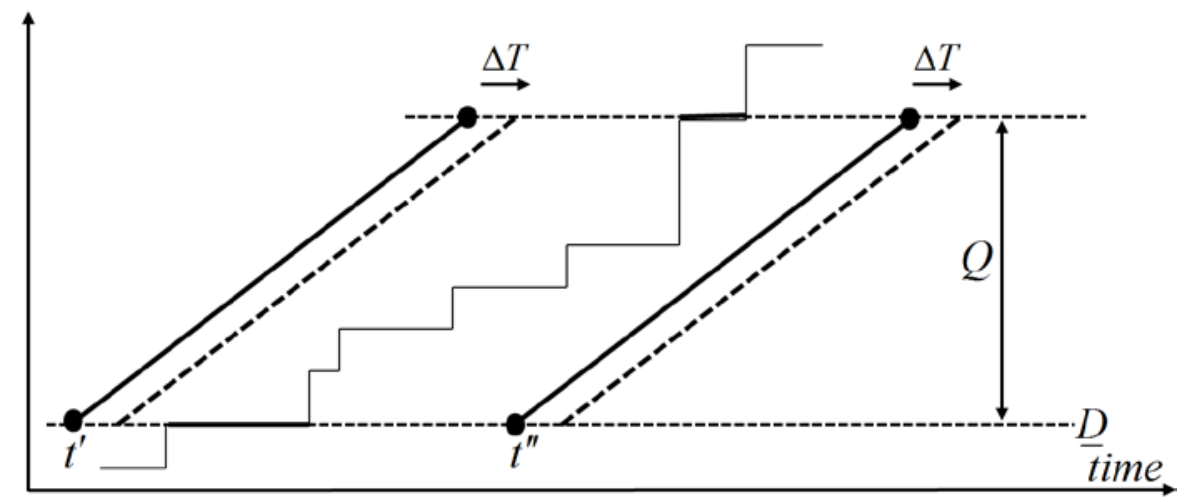

Figure 7. Moving a production ramp to the left or right of the demand staircase.

We first find that the optimal ramp is confined to a time interval, as stated in:

\section{Lemma 1}

With a profitable batch, the optimal timing of the ramp must be found in the interval between the leftmost ramp touching the demand staircase and the similar rightmost ramp.

These points in time are denoted $t_{v}$ and $t_{w}$ as shown below in Figure 8, and are defined from the points of tangency $A$ and $B$.

\section{Proof}

Consider Figure 7. If the batch is produced beginning at $t^{\prime}$ to the left in the figure not touching the demand staircase and a rightward move is made by $\Delta T$, the resulting change in $\mathrm{NPV}$ will be $\Delta \mathrm{NPV}=e^{-\rho t^{\prime}}\left(1-e^{-\rho \Delta T}\right)\left((c q / \rho)\left(1-e^{-\rho Q / q}\right)+K e^{-\rho \beta Q / q}\right)$, which obviously is positive. Here, as before $\beta$ is zero, if the setup cost occurs at the beginning of the ramp (at $t^{\prime}$ ) and unity if it located to the end (at $t^{\prime}+Q / q$ ). On the other hand, if the ramp is to the right of the demand staircase beginning at $t^{\prime \prime}$ (as shown in the right part of Figure 7), a move further right by $\Delta T$ will change the NPV by $\triangle \mathrm{NPV}=$ $-e^{-\rho t^{\prime \prime}}\left(1-e^{-\rho \Delta T}\right)\left(((p-c) q / \rho)\left(1-e^{-\rho Q / q}\right)-K e^{-\rho \beta Q / q}\right)$. If the batch is profitable, meaning that the profit margin $(p-c)$ is high enough to make the NPV of the batch positive, $\left(((p-c) q / \rho)\left(1-e^{-\rho Q / q}\right)-K e^{-\rho \beta Q / q}\right)>0$, a leftward move is profitable.

Hence, with a profitable batch, the optimal timing of the ramp must be found in the interval between $t_{v}$ and $t_{w}$. 


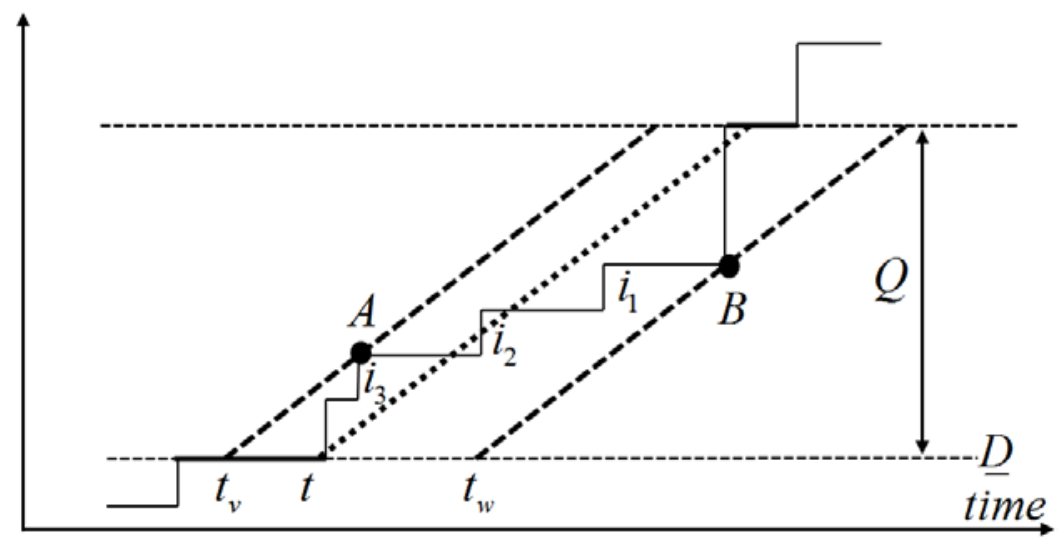

Figure 8. The production ramp intersecting the demand staircase.

Let us now examine how the NPV behaves when the ramp starts at an intermediate time, say $t$, $t_{v} \leq t \leq t_{w}$, i.e. the dotted ramp in Figure 8. Following this ramp upwards, it covers consecutive steps of demand $D_{i}, i=i_{\min }, i_{\min }+1, \ldots, i_{\max }$, with $i_{\min } \leq i_{\max }, \bar{D}_{i_{\min }-1}=\underline{D}$, $\bar{D}_{i_{\max }}=\underline{D}+Q$.

We distinguish three subsets of the set of indices $S=\left\{i_{\min }, \ldots, i_{\max }\right\}$, which are denoted $\mathrm{S}_{1}, \mathrm{~S}_{2}, \mathrm{~S}_{3}$, and where $\mathrm{S}=\mathrm{S}_{1} \cup \mathrm{S}_{2} \cup \mathrm{S}_{3}$. Either an index represents a demand step $D_{i}$ below the ramp (dotted, beginning at $t$ in Figure 8) and then $i \in \mathrm{S}_{1}$ (such as $i_{1}$ in the figure), or demand and production cross each other and $i \in S_{2}$ ( $i_{2}$ in the figure), or the step is above the ramp $i \in \mathrm{S}_{3}$ ( $i_{3}$ in the figure). If the ramp happens to touch a corner, the corresponding index may belong to either of two subsets, but below the NPV as a function of time $t$ is shown to be continuous, and it then does not matter which subset is chosen in such a case. We let $\mathrm{S}_{1}$ contains indices satisfying $q\left(t_{i}-t\right) \geq\left(\bar{D}_{i}-\underline{D}\right) \quad, \quad \mathrm{S}_{2} \quad$ indices satisfying $\left(\bar{D}_{i-1}-\underline{D}\right) \leq q\left(t_{i}-t\right)<\left(\bar{D}_{i}-\underline{D}\right)$, and $\mathrm{S}_{3}$ indices satisfying $q\left(t_{i}-t\right)<\left(\bar{D}_{i-1}-\underline{D}\right)$.

The contribution to the NPV of the batch from one of the included steps $D_{i}$ depends on which of the three subsets to which its index $i$ belongs.

The setup cost contribution is common for the whole batch and will be $-K e^{-\rho(t+\beta Q / q)}$. Irrespective of the subset to which $i$ belongs, the contribution to the NPV from production costs during step $i$ will be $-(c q / \rho)\left(1-e^{-\rho D_{i} / q}\right) e^{-\rho\left(t+\left(\bar{D}_{i-1}-\underline{D}\right) / q\right)}$. The contribution from revenues will be $p D_{i} e^{-\rho t_{i}}$ if $i \in \mathrm{S}_{1}$ (all $D_{i}$ units are sold at $t_{i}$ ), $p\left(\left(t_{i}-t\right) q-\left(\bar{D}_{i-1}-\underline{D}\right)\right) e^{-\rho t_{i}}+(p q / \rho)\left(1-e^{-\rho\left(t+\left(\bar{D}_{i}-\underline{D}\right) / q-t_{i}\right)}\right) e^{-\rho t_{i}}, \quad$ if $\quad i \in \mathrm{S}_{2}$ (meaning that $\left(t_{i}-t\right) q-\left(\bar{D}_{i-1}-\underline{D}\right)$ units are sold at $t_{i}$, and the remainder backlogged and sold as production provides these units as an output). Finally, if $i \in \mathrm{S}_{3}$ then all $D_{i}$ units will be backlogged and the NPV of revenues from the step becomes $(p q / \rho)\left(1-e^{-\rho D_{i} / q}\right) e^{-\rho\left(\bar{D}_{i-1}-\underline{D}\right) / q} e^{-\rho t}$. 
If the ramp touches an upper corner at $t_{i}$, we have $t=t_{i}-\left(\bar{D}_{i}-\underline{D}\right) / q$ and the revenue contribution will be $p D_{i} e^{-\rho t_{i}}$, either $i$ is considered as belonging to $\mathrm{S}_{1}$ or to $\mathrm{S}_{2}$, and if the ramp touches a lower corner at $t_{i}$ then $t=t_{i}-\left(\bar{D}_{i-1}-\underline{D}\right) / q$ and the revenue contribution becomes $(p q / \rho)\left(1-e^{-\rho D_{i} / q}\right) e^{-\rho t_{i}}$, irrespective of whether $i$ is considered belonging to $\mathrm{S}_{2}$ or to $\mathrm{S}_{3}$.

This implies that the NPV of the whole batch is a continuous function of time as $t$ increases and the ramp passes through the demand staircase and crosses different corners. As shown below, also the first time derivative of NPV is continuous as corners are passed. However, this is not so for the second time derivative, which indeed makes jumps at the corners.

We thus conclude that the NPV of a batch discounted to time zero may be written as the following sum:

$$
\begin{gathered}
\mathrm{NPV}_{\text {batch }}(t)=-(c q / \rho) \sum_{i \in \mathrm{S}}\left(1-e^{-\rho D_{i} / q}\right) e^{-\rho\left(t+\left(\bar{D}_{i-1}-\underline{D}\right) / q\right)}-K e^{-\rho(t+\beta Q / q)}+p \sum_{i \in \mathrm{S}_{1}} D_{i} e^{-\rho t_{i}} \\
+(p q / \rho) \sum_{i \in \mathrm{S}_{2}} e^{-\rho t_{i}}\left(\rho\left(\left(t_{i}-t\right)-\left(\bar{D}_{i-1}-\underline{D}\right) / q\right)+\left(1-e^{-\rho\left(t-t_{i}+\left(\bar{D}_{i}-\underline{D}\right) / q\right)}\right)\right) \\
+(p q / \rho) e^{-\rho t} \sum_{i \in S_{3}}\left(1-e^{-\rho D_{i} / q}\right) e^{-\rho\left(\bar{D}_{i-1}-\underline{D}\right) / q}
\end{gathered}
$$

The slope of $\mathrm{NPV}_{\text {batch }}(t)$ as the ramp passes different corners is now examined. The first and second time derivatives of $\mathrm{NPV}_{\text {batch }}(t)$ are derived as:

$$
\begin{gathered}
\frac{d \mathrm{NPV}_{\text {batch }}(t)}{d t}=c q \sum_{i \in \mathrm{S}}\left(1-e^{-\rho D_{i} / q}\right) e^{-\rho\left(t+\left(\bar{D}_{i-1}-\underline{D}\right) / q\right)}+\rho K e^{-\rho(t+\beta Q / q)} \\
-p q \sum_{i \in \mathrm{S}_{2}} e^{-\rho t_{i}}\left(1-e^{-\rho\left(t-t_{i}+\left(\bar{D}_{i}-\underline{D}\right) / q\right)}\right)-p q e^{-\rho t} \sum_{i \in \mathrm{S}_{3}}\left(1-e^{-\rho D_{i} / q}\right) e^{-\rho\left(\bar{D}_{i-1}-\underline{D}\right) / q}, \\
\frac{d^{2} \mathrm{NPV}_{\text {batch }}(t)}{d t^{2}}=-\rho e^{-\rho t}\left(c q \sum_{i \in \mathrm{S}}\left(1-e^{-\rho D_{i} / q}\right) e^{-\rho\left(\bar{D}_{i-1}-\underline{D}\right) / q}+\rho K e^{-\rho \beta Q / q}\right. \\
\left.+p q \sum_{i \in S_{2}} e^{-\rho\left(\bar{D}_{i}-\underline{D}\right) / q}-p q \sum_{i \in \mathrm{S}_{3}}\left(1-e^{-\rho D_{i} / q}\right) e^{-\rho\left(\bar{D}_{i-1}-\underline{D}\right) / q}\right) .
\end{gathered}
$$

At each $t_{i}$ there are two corners: one upper and one lower corner. We let $\left[\frac{d \mathrm{NPV}_{\text {batch }}(t)}{d t}\right]_{i, \text { upper }}^{-}$ denote the derivative immediately before the upper corner is touched, and $\left[\frac{d \mathrm{NPV}_{\text {batch }}(t)}{d t}\right]_{i, \text { upper }}^{+}$ immediately after, with a similar notation at lower corners and for the second time derivative. At an upper corner at $t_{i}$ we have $t=t_{i}-\left(\bar{D}_{i}-\underline{D}\right) / q$, and at a lower corner $t=t_{i}-\left(\bar{D}_{i-1}-\underline{D}\right) / q$. 
We now show that:

\section{Lemma 2}

The time derivative of $\mathrm{NPV}_{\text {batch }}(t)$ is continuous.

\section{Proof}

Since the NPV of production and setup costs obviously are continuous, it suffices to examine contributions from the revenue terms. Before passing an upper corner at $t_{k}, k$ belongs to $\mathrm{S}_{1}$, and after passing, $k$ belongs to $S_{2}$. So, from (5) we have

$$
\begin{gathered}
{\left[\frac{d \mathrm{NPV}_{\text {batch }}(t)}{d t}\right]_{k, \text { upper }}^{+}-\left[\frac{d \mathrm{NPV}_{\text {batch }}(t)}{d t}\right]_{k, \text { upper }}^{-}=} \\
=-p q \sum_{\substack{i \in \mathrm{S}_{2}, k \notin \mathrm{S}_{2}}} e^{-\rho t_{i}}\left(1-e^{-\rho\left(t-t_{i}+\left(\bar{D}_{i}-\underline{D}\right) / q\right)}\right)+p q \sum_{i, k \in \mathrm{S}_{2}} e^{-\rho t_{i}}\left(1-e^{-\rho\left(t-t_{i}+\left(\bar{D}_{i}-\underline{D}\right) / q\right)}\right)= \\
=p q e^{-\rho t_{k}}\left(1-e^{-\rho\left(t-t_{k}+\left(\bar{D}_{k}-\underline{D}\right) / q\right)}\right)=0,
\end{gathered}
$$

since $t=t_{k}-\left(\bar{D}_{k}-\underline{D}\right) / q$ at the upper corner at $t_{k}$. Similarly, for a lower corner at $t_{k}$, before the passing, $k$ belongs to $\mathrm{S}_{2}$, and after, $k$ belongs to $\mathrm{S}_{3}$. Therefore,

$$
\begin{gathered}
{\left[\frac{d \mathrm{NPV}_{\text {batch }}(t)}{d t}\right]_{k, \text { lower }}^{+}-\left[\frac{d \mathrm{NPV}_{\text {batch }}(t)}{d t}\right]_{k, \text { lower }}^{-}=} \\
=-p q \sum_{i, k \in \mathrm{S}_{2}} e^{-\rho t_{i}}\left(1-e^{-\rho\left(t-t_{i}+\left(\bar{D}_{i}-\underline{D}\right) / q\right)}\right)-p q e^{-\rho t} \sum_{\substack{i \in S_{3}, k \notin S_{3}}}\left(1-e^{-\rho D_{i} / q}\right) e^{-\rho\left(\bar{D}_{i-1}-\underline{D}\right) / q} \\
+p q \sum_{\substack{i \in S_{2}, k \notin \mathcal{S}_{2}}} e^{-\rho t_{i}}\left(1-e^{-\rho\left(t-t_{i}+\left(\bar{D}_{i}-\underline{D}\right) / q\right)}\right)+p q e^{-\rho t} \sum_{i, k \in S_{3},}\left(1-e^{-\rho D_{i} / q}\right) e^{-\rho\left(\bar{D}_{i-1}-\underline{D}\right) / q}= \\
=-p q e^{-\rho t_{k}}\left(1-e^{-\rho\left(t-t_{k}+\left(\bar{D}_{k}-\underline{D}\right) / q\right)}\right)+p q e^{-\rho t}\left(1-e^{-\rho D_{k} / q}\right) e^{-\rho\left(\bar{D}_{k-1}-\underline{D}\right) / q}=0,
\end{gathered}
$$

since $t=t_{k}-\left(\bar{D}_{k-1}-\underline{D}\right) / q$ at the lower corner and $\bar{D}_{k}=\bar{D}_{k-1}+D_{k}$.

Hence the first time derivative is continuous.

Concerning the second time derivative, we state the following:

\section{Lemma 3}

When a ramp moves from left to right, the second time derivative of the NPV of the batch is increased by a positive amount $\rho p q e^{-\rho t_{k}}$, when passing the upper corner at $t_{k}$, and is decreased by the same amount, when later passing the lower corner at $t_{k}$. 


\section{Proof}

Let us examine the difference before and passing an upper corner at $t_{k}$. From (6) with $t=t_{k}-\left(\bar{D}_{k}-\underline{D}\right) / q$, we then have

$$
\begin{gathered}
{\left[\frac{d^{2} \mathrm{NPV}_{\text {batch }}(t)}{d t^{2}}\right]_{k, \text { upper }}^{+}-\left[\frac{d^{2} \mathrm{NPV}_{\text {batch }}(t)}{d t^{2}}\right]_{k, \text { upper }}^{-}=} \\
=-p q \rho e^{-\rho t}\left(\sum_{\substack{i \in S_{2}, k \notin \mathcal{S}_{2}}} e^{-\rho\left(\bar{D}_{i}-\underline{D}\right) / q}-\sum_{i, l \in \mathcal{S}_{2}} e^{-\rho\left(\bar{D}_{i}-\underline{D}\right) / q}\right)=\rho p q e^{-\rho t_{k}}>0 .
\end{gathered}
$$

Instead at the lower corner of $t_{k}$, where $t=t_{k}-\left(\bar{D}_{k-1}-\underline{D}\right) / q$ and $k$ moves from $\mathrm{S}_{2}$ to $\mathrm{S}_{3}$, we have

$$
\begin{aligned}
& {\left[\frac{d^{2} \mathrm{NPV}_{\text {batch }}(t)}{d t^{2}}\right]_{k, \text { lower }}^{+}-\left[\frac{d^{2} \mathrm{NPV}_{\text {batch }}(t)}{d t^{2}}\right]_{k, \text { lower }}^{-}=} \\
& =-\rho e^{-\rho t}\left(p q \sum_{i, k \in S_{2},} e^{-\rho\left(\bar{D}_{i}-\underline{D}\right) / q}-p q \sum_{\substack{i \in S_{3}, k \notin S_{3}}}\left(1-e^{-\rho D_{i} / q}\right) e^{-\rho\left(\bar{D}_{i-1}-\underline{D}\right) / q}\right) \\
& +\rho e^{-\rho t}\left(p q \sum_{\substack{i \in S_{S^{\prime}}, k \notin S_{2}}} e^{-\rho\left(\bar{D}_{i}-\underline{D}\right) / q}-p q \sum_{i, k \in S_{3}}\left(1-e^{-\rho D_{i} / q}\right) e^{-\rho\left(\bar{D}_{i-1}-\underline{D}\right) / q}\right) \\
& =-\rho p q e^{-\rho t_{k}}<0 \text {. }
\end{aligned}
$$

Hence we conclude that the second derivative steps upwards by $\rho p q e^{-\rho t_{k}}$ at an upper corner, and makes an equally-sized downward step at the lower corner.

Thus the effects of the pairs of discontinuities cancel as the complete demand staircase has been passed. The dampening effect from the discount factor $e^{-\rho t_{k}}$, shows that the up- and downward steps diminish in size, when the ramp moves rightwards from one step to the next.

To the very left, when the first upper corner is touched ( $A$ in Figure 8), where $S_{2}$ and $S_{3}$ are empty, so $\mathrm{S}=\mathrm{S}_{1}$, the second derivative at the left of this point is $\left[\frac{d^{2} \mathrm{NPV}_{\text {batch }}}{d t^{2}}\right]_{v, \text { upper }}^{-}=-\rho e^{-\rho t}\left(c q \sum_{i \in \mathrm{S}}\left(1-e^{-\rho D_{i} / q}\right) e^{-\rho\left(\bar{D}_{i-1}-\underline{D}\right) / q}+\rho K e^{-\rho \beta Q / q}\right)<0 \quad$, whereas it becomes $\left[\frac{d^{2} \mathrm{NPV}_{\text {batch }}}{d t^{2}}\right]_{w, \text { lower }}^{+}=\rho e^{-\rho t}\left((p-c) q\left(1-e^{-\rho Q / q}\right)-\rho K e^{-\rho \beta Q / q}\right)>0$ immediately after having passed the rightmost lower corner ( $B$ in Figure 8), at which point $S_{1}$ and $S_{2}$ are empty, so $\mathrm{S}=\mathrm{S}_{3}$, assuming the batch to be profitable.

So the NPV function of a profitable batch starts at $t_{v}$ as concave and ends at $t_{w}$ as convex. 
Immediately after having passing the leftmost upper corner at $A$ in Figure 8 at $t_{v}$, then $t=t_{v}-\left(\bar{D}_{v}-\underline{D}\right) / q$. Here $\mathrm{S}_{1}$ will contain all indices except $v, \mathrm{~S}_{2}$ will contain only $v$, and $\mathrm{S}_{3}$ will be empty. The derivative at this point is

$$
\left[\frac{d \mathrm{NPV}_{\text {batch }}}{d t}\right]_{v, \text { upper }}^{+}=e^{-\rho\left(t_{v}-\left(\bar{D}_{v}-\underline{D}\right) / q\right)}\left(c q \sum_{i \in \mathrm{Se}}\left(1-e^{-\rho D_{v} / q}\right) e^{-\rho\left(\bar{D}_{v-1}-\underline{D}\right) / q}+\rho K e^{-\rho \beta Q / q}\right)>0,
$$

since the single term for $v$ in $\mathrm{S}_{2}$ disappears for this value of $t$. Hence $\mathrm{NPV}_{\text {batch }}(t)$ is increasing as the ramp has just hit the leftmost corner.

Similarly, just before leaving the rightmost (lower) corner at $t_{w}$ ( $B$ in Figure 8), here $t=t_{w}-\left(\bar{D}_{w-1}-\underline{D}\right) / q, \mathrm{~S}_{2}$ contains only $w$, and $\mathrm{S}_{3}$ contains all indices except $w$. The derivative at that corner then is

$$
\begin{gathered}
{\left[\frac{d \mathrm{NPV}_{\text {batch }}}{d t}\right]_{w, \text { lower }}^{-}=e^{-\rho\left(t_{w}-\left(\bar{D}_{w-1}-\underline{D}\right) / q\right)}\left(c q \sum_{i \in \mathrm{S}}\left(1-e^{-\rho D_{i} / q}\right) e^{-\rho\left(\bar{D}_{i-1}-\underline{D}\right) / q}+\rho K e^{-\rho \beta Q / q}\right)} \\
-p q e^{-\rho t_{w}}\left(1-e^{-\rho\left(t-t_{w}+\left(\bar{D}_{w}-\underline{D}\right) / q\right)}\right)-p q e^{-\rho\left(t_{w}-\left(\bar{D}_{w-1}-\underline{D}\right) / q\right)} \sum_{i \in S, i \neq w}\left(1-e^{-\rho D_{i} / q}\right) e^{-\rho\left(\bar{D}_{i-1}-\underline{D}\right) / q}= \\
=-e^{-\rho\left(t_{w}-\left(\bar{D}_{w-1}-\underline{D}\right) / q\right)}\left((p-c) q\left(1-e^{-\rho Q / q}\right)-\rho K e^{-\rho \beta Q / q}\right)<0,
\end{gathered}
$$

which is negative, if the batch is profitable. In the developments of (12), the single tern in $S_{2}$ cancels with the missing term in $\mathrm{S}$, and the summation $\sum_{i \in \mathrm{S}}\left(1-e^{-\rho D_{i} / q}\right) e^{-\rho\left(\bar{D}_{i-1}-\underline{D}\right) / q}=\left(1-e^{-\rho Q / q}\right)$ is made use of.

Hence for a profitable batch, its NPV starts at $t_{v}$ by increasing in a concave fashion and ends at $t_{w}$ by decreasing in a convex fashion.

hit

\section{Lemma 4}

When moving the production ramp along the time scale from upper corner to next upper corner, the time derivative of $\mathrm{NPV}_{\text {batch }}(t)$ is positive at a first sequence of corners, after which it becomes negative for the remaining sequence of corners. The same result obtains when moving between lower corners.

\section{Proof}

Upper corners

Assuming that only one corner is passed at a time (avoiding the unlikely case of two or more corners being on a slope equal to that of the ramp), we investigate the change in the value of the derivative, when passing from one upper corner to the closest next upper corner. Assume that these corners are at $t_{k}$ and $t_{m}, m>l$, and that the derivatives are evaluated immediately after passing. At the first corner $\mathrm{S}_{1}$ contains $m$ and $\mathrm{S}_{2}$ contains $k$, and $\mathrm{S}_{3}$ neither contains $k$ nor $m$. At this corner, where $t=t_{k}-\left(\bar{D}_{k}-\underline{D}\right) / q$, from (5) we have 


$$
\begin{gathered}
{\left[\frac{d \mathrm{NPV}_{\text {batch }}(t)}{d t}\right]_{k, \text { upper }}^{+}=q e^{-\rho\left(t_{k}-\left(\bar{D}_{k}-\underline{D}\right) / q\right)}\left(c \sum_{i \in \mathrm{S}}\left(1-e^{-\rho D_{i} / q}\right) e^{-\rho\left(\bar{D}_{i-1}-\underline{D}\right) / q}+(\rho K / q) e^{-\rho \beta Q / q}\right.} \\
\left.-p \sum_{\substack{i, k \in S_{2}, m \notin \mathrm{S}_{2}}}\left(e^{\rho\left(t_{k}-t_{i}-\left(\bar{D}_{k}-\underline{D}\right) / q\right)}-e^{-\rho\left(\bar{D}_{i}-\underline{D}\right) / q}\right)-p \sum_{\substack{i \in S_{3}, k, m \notin S_{3}}}\left(1-e^{-\rho D_{i} / q}\right) e^{-\rho\left(\bar{D}_{i-1}-\underline{D}\right) / q}\right)= \\
=q e^{-\rho\left(t_{k}-\left(\bar{D}_{k}-\underline{D}\right) / q\right)} A_{k},
\end{gathered}
$$

where the abbreviation $A_{k}$ is defined by the expression within the parenthesis.

At the later upper corner at $t_{m}, \mathrm{~S}_{2}$ contains the newcomer $m$, whereas $\mathrm{S}_{2}$ might still contain $k$ (if the corners are close), or $k$ might have moved to $S_{3}$ (if the corners are more distant). If $k$ still belongs to $S_{2}$, then the slope of $\operatorname{NPV}_{\text {batch }}(t)$ becomes

$$
\begin{aligned}
& {\left[\frac{d \mathrm{NPV}_{\text {batch }}(t)}{d t}\right]_{m, \text { upper }}^{+}=} \\
& =q e^{-\rho\left(t_{m}-\left(\bar{D}_{m}-\underline{D}\right) / q\right)}\left(c \sum_{i \in S}\left(1-e^{-\rho D_{i} / q}\right) e^{-\rho\left(\bar{D}_{i-1}-\underline{D}\right) / q}+(\rho K / q) e^{-\rho \beta Q / q}\right. \\
& \left.-p \sum_{\substack{i \in S_{2}, k, m \in S_{2}}}\left(e^{\rho\left(t_{m}-t_{i}-\left(\bar{D}_{m}-\underline{D}\right) / q\right)}-e^{-\rho\left(\bar{D}_{i}-\underline{D}\right) / q}\right)-p \sum_{\substack{i \in S_{3}, k, m \notin S_{3}}}\left(1-e^{-\rho D_{i} / q}\right) e^{-\rho\left(\bar{D}_{i-1}-\underline{D}\right) / q}\right)=q e^{-\rho\left(t_{m}-\left(\bar{D}_{m}-\underline{D}\right) / q\right)} A_{m}^{\prime},
\end{aligned}
$$

where the abbreviation $A_{m}^{\prime}$ is defined by the expression within the parenthesis and the prime refers to the case of the two corners being close. If, instead in the more distant case, $k$ has moved from $\mathrm{S}_{2}$ to $\mathrm{S}_{3}$, the slope at upper corner $t_{m}$ is

$$
\begin{aligned}
& {\left[\frac{d \mathrm{NPV}_{\text {batch }}}{d t}\right]_{m, \text { upper }}^{+}=} \\
& =q e^{-\rho\left(t_{m}-\left(\bar{D}_{m}-\underline{D}\right) / q\right)}\left(C \sum_{i \in \mathrm{S}}\left(1-e^{-\rho D_{i} / q}\right) e^{-\rho\left(\bar{D}_{i-1}-\underline{D}\right) / q}+(\rho K / q) e^{-\rho \beta Q / q}\right. \\
& \left.-p \sum_{\substack{i, m \in S_{2}, k \notin S_{2}}}\left(e^{\rho\left(t_{m}-t_{i}-\left(\bar{D}_{m}-\underline{D}\right) / q\right)}-e^{-\rho\left(\bar{D}_{i}-\underline{\underline{D}}\right) / q}\right)-p \sum_{\substack{i, k \in \mathcal{S}_{\mathcal{S}_{3}}, m \notin S_{3}}}\left(1-e^{-\rho D_{i} / q}\right) e^{-\rho\left(\bar{D}_{i-1}-\underline{D}\right) / q}\right)=q e^{-\rho\left(t_{m}-\left(\bar{D}_{m}-\underline{D}\right) / q\right)} A_{m}^{\prime \prime},
\end{aligned}
$$

with $A_{m}^{\prime \prime}$ similarly defined, but now referring to the case of distant points.

We examine the differences between $A_{1}$ and $A_{m}^{\prime}, A_{m}^{\prime \prime}$, respectively. Using the expressions in (13), (14) and (15), we obtain

$$
A_{m}^{\prime}-A_{k}=0
$$




$$
A_{m}^{\prime \prime}-A_{k}=-p\left(1-e^{-\rho D_{k} / q}\right) e^{-\rho\left(\bar{D}_{k-1}-\underline{D}\right) / q}<0
$$

Hence we conclude that if at the later point $A_{m}^{\prime}>0$, then at the earlier point we must have $A_{k}>0$, and if $A_{k}<0$ at the earlier point, then we must have $A_{k}^{\prime}<0$, and, similarly, if $A_{m}^{\prime \prime}>0$ then $A_{k}>0$, and if $A_{k}<0$ then $A_{m}^{\prime \prime}<0$, depending on which case obtains.

The derivatives at the two points have the same sign as $A_{k}$ and $A_{m}^{\prime}$, $A_{m}^{\prime \prime}$, respectively, so an increase in $\mathrm{NPV}_{\text {batch }}(t)$ at a corner must be preceded by an increase, and a decrease must be succeeded by a decrease.

\section{Lower corners}

Since the derivative is continuous, we allocate the index of a lower corner to $S_{2}$, when the ramp touches that corner. When moving from one lower corner at $t_{k}$ to a next later lower corner at $t_{m}, k$ leaves from $S_{2}$ to $S_{3}$, and either $m$ moves from $S_{1}$ to $S_{2}$ or $m$ stays in $S_{2}$.

If $m$ moves from $\mathrm{S}_{1}$ to $\mathrm{S}_{2}$ the derivatives are

$$
\begin{aligned}
& {\left[\frac{d \mathrm{NPV}_{\text {batch }}(t)}{d t}\right]_{k, \text { lower }}^{-}=e^{-\rho\left(t_{k}-\left(\bar{D}_{k-1}-\underline{D}\right) / q\right)}\left(c q \sum_{i \in \mathrm{S}}\left(1-e^{-\rho D_{i} / q}\right) e^{-\rho\left(\bar{D}_{i-1}-\underline{D}\right) / q}+\rho K e^{-\rho \beta Q / q}\right.} \\
& \left.-p q \sum_{\substack{i, k \in \mathcal{S}_{2}, m \notin S_{2}}}\left(e^{\rho\left(t_{k}-t_{i}-\left(\bar{D}_{k-1}-\underline{D}\right) / q\right)}-e^{-\rho\left(\bar{D}_{i}-\underline{D}\right) / q}\right)-p q \sum_{\substack{i \in \mathcal{S}_{2}, k, m \notin S_{3}}}\left(1-e^{-\rho D_{i} / q}\right) e^{-\rho\left(\bar{D}_{i-1}-\underline{D}\right) / q}\right)=e^{-\rho\left(t_{k}-\left(\bar{D}_{k-1}-\underline{D}\right) / q\right)} B_{k}, \\
& {\left[\frac{d \mathrm{NPV}_{\text {batch }}(t)}{d t}\right]_{m, \text { lower }}^{-}=e^{-\rho\left(t_{m}-\left(\bar{D}_{m-1}-\underline{D}\right) / q\right)}\left(c q \sum_{i \in \mathrm{S}}\left(1-e^{-\rho D_{i} / q}\right) e^{-\rho\left(\bar{D}_{i-1}-\underline{D}\right) / q}+\rho K e^{-\rho \beta Q / q}\right.} \\
& \left.-p q \sum_{\substack{i, m \in \mathcal{S}_{2} \\
k \notin S_{2}}}\left(e^{\rho\left(t_{m}-t_{i}-\left(\bar{D}_{m-1}-\underline{D}\right) / q\right)}-e^{-\rho\left(\bar{D}_{i}-\underline{D}\right) / q}\right)-p q \sum_{\substack{i, k \in S_{3}, m \notin S_{3}}}\left(1-e^{-\rho D_{i} / q}\right) e^{-\rho\left(\bar{D}_{i-1}-\underline{D}\right) / q}\right)=e^{-\rho\left(t_{m}-\left(\bar{D}_{m-1}-\underline{D}\right) / q\right)} B_{m},
\end{aligned}
$$

where the abbreviations $B_{k}$ and $B_{m}$ are defined by the expressions within the parentheses. The difference in this case is computed as $B_{m}-B_{k}=-p q e^{-\rho\left(\bar{D}_{m-1}-\underline{D}\right) / q}\left(1-e^{-\rho D_{m} / q}\right)<0$. When instead $m$ stays in $\mathrm{S}_{2}$, we have

$$
\begin{gathered}
{\left[\frac{d \mathrm{NPV}_{\text {batch }}(t)}{d t}\right]_{k, \text { lower }}^{-}=e^{-\rho\left(t_{k}-\left(\bar{D}_{k-1}-\underline{D}\right) / q\right)}\left(c q \sum_{i \in \mathrm{S}}\left(1-e^{-\rho D_{i} / q}\right) e^{-\rho\left(\bar{D}_{i-1}-\underline{D}\right) / q}+\rho K e^{-\rho \beta Q / q}\right.} \\
\left.-p q \sum_{i, k, m \in \mathrm{S}_{2},}\left(e^{\rho\left(t_{k}-t_{i}-\left(\bar{D}_{k-1}-\underline{D}\right) / q\right)}-e^{-\rho\left(\bar{D}_{i}-\underline{D}\right) / q}\right)-p q \sum_{\substack{i \in S_{3}, k, m \notin S_{3}}}\left(1-e^{-\rho D_{i} / q}\right) e^{-\rho\left(\bar{D}_{i-1}-\underline{D}\right) / q}\right)=e^{-\rho\left(t_{k}-\left(\bar{D}_{k-1}-\underline{D}\right) / q\right)} B_{k},
\end{gathered}
$$




$$
\begin{aligned}
& {\left[\frac{d \mathrm{NPV}_{\text {batch }}(t)}{d t}\right]_{m, \text { lower }}^{-}=e^{-\rho\left(t_{m}-\left(\bar{D}_{m-1}-\underline{D}\right) / q\right)}\left(c q \sum_{i \in \mathrm{S}}\left(1-e^{-\rho D_{i} / q}\right) e^{-\rho\left(\bar{D}_{i-1}-\underline{D}\right) / q}+\rho K e^{-\rho \beta Q / q}\right.} \\
& \left.-p q \sum_{\substack{i, m \in S_{2} \\
k \notin S_{2}}}\left(e^{\rho\left(t_{m}-t_{i}-\left(\bar{D}_{m-1}-\underline{D}\right) / q\right)}-e^{-\rho\left(\bar{D}_{i}-\underline{D}\right) / q}\right)-p q \sum_{\substack{i, k \in S_{3}, m \notin S_{3}}}\left(1-e^{-\rho D_{i} / q}\right) e^{-\rho\left(\bar{D}_{i-1}-\underline{D}\right) / q}\right)=e^{-\rho\left(t_{m}-\left(\bar{D}_{m-1}-\underline{D}\right) / q\right)} B_{m},
\end{aligned}
$$

and the difference becomes zero, $B_{m}-B_{k}=0$.

So we can draw the same conclusion as for upper corners.

That $\mathrm{NPV}_{\text {batch }}(t)$ is increasing at a left sequence of corners, and then decreasing at the remaining sequence of corners, indicates that the function is unimodal, but does not prove this. This question is now investigated leading to the main result of

\section{Theorem 2}

The NPV of a batch has a unique maximum.

\section{Proof}

We know from earlier lemmas that a maximum must be found between $t_{v}$ and $t_{w}$, which means that the set $S_{2}$ is non-empty at the optimum.

From (4), (5) and (6) by taking weighted sums of $\mathrm{NPV}_{\text {batch }}(t)$ and $d \mathrm{NPV}_{\text {batch }} / d t$, and of $d \mathrm{NPV}_{\text {batch }} / d t$ and $d^{2} \mathrm{NPV}_{\text {batch }} / d t^{2}$, respectively, we find the simple relations:

$$
\rho \mathrm{NPV}_{\text {batch }}(t)+\frac{d \mathrm{NPV}_{\text {batch }}}{d t}=p \rho\left(\sum_{i \in \mathrm{S}_{1}} D_{i} e^{-\rho t_{i}}+q \sum_{i \in \mathrm{S}_{2}} e^{-\rho t_{i}}\left(t_{i}-t-\left(\bar{D}_{i-1}-\underline{D}\right) / q\right)\right) \geq 0 \text {, }
$$

which is non-negative since $\left(t_{i}-t-\left(\bar{D}_{i-1}-\underline{D}\right) / q\right) \geq 0$ for $i \in \mathrm{S}_{2}$, and similarly,

$$
\rho \frac{d \mathrm{NPV}_{\mathrm{batch}}}{d t}+\frac{d^{2} \mathrm{NPV}_{\mathrm{batch}}}{d t^{2}}=-\rho p q \sum_{i \in \mathrm{S}_{2}} e^{-\rho t_{i}} \leq 0
$$

At a local interior extreme point $\frac{d \mathrm{NPV}_{\text {batch }}}{d t}=0$, there $\frac{d^{2} \mathrm{NPV}_{\text {batch }}}{d t^{2}}<0$, if the set $\mathrm{S}_{2}$ is nonempty. But if it is empty, then either we are to the right of $t_{w}$ or to the left of $t_{v}$. So the sign of $\frac{d \mathrm{NPV}_{\text {batch }}}{d t}$ cannot change more than once and indeed this is a maximum. If the maximum would have been hypothesised to be at a pointed corner, this would contradict the continuity property of the first derivative.

To the right of $t_{w}$ and to the left of $t_{v}$, where $\mathrm{S}_{2}$ is empty, $\frac{d^{2} \mathrm{NPV}_{\text {batch }}}{d t^{2}}$ and $\rho \cdot \frac{d \mathrm{NPV}_{\text {batch }}}{d t}$ are mirror images of each other, as well as of $\rho^{2} \cdot \mathrm{NPV}_{\text {batch }}$. 
To the left of $t_{v} \mathrm{NPV}_{\text {batch }}$ and $\frac{d \mathrm{NPV}_{\text {batch }}}{d t}=0$ follow each other at a distance, with $\mathrm{NPV}_{\text {batch }}$ above, since $\mathrm{S}_{1}$ is non-empty (it includes all steps). To the right of $t_{w}$ where both $\mathrm{S}_{1}$ and $\mathrm{S}_{2}$ are empty, $\rho^{2} \mathrm{NPV}_{\text {batch }}(t)$ and $\frac{d^{2} \mathrm{NPV}_{\text {batch }}(t)}{d t^{2}}$ follow each other perfectly (compare Figures 12 and 13 below).

At the optimum, where $\frac{d N P V_{\text {batch }}}{d t}=0$, we have

$$
\mathrm{NPV}_{\text {batch }}(t)=p\left(\sum_{i \in \mathrm{S}_{1}} D_{i} e^{-\rho t_{i}}+q \sum_{i \in \mathrm{S}_{2}} e^{-\rho t_{i}}\left(t_{i}-t-\left(\bar{D}_{i-1}-\underline{D}\right) / q\right)\right) \text {. }
$$

which also shows the maximum to be positive. It may be interesting to note that the expression contains neither the setup cost parameter $K$, nor the production cost parameter $c$. However, the optimum value of $t$, which is present here, certainly depends on all parameters.

The discussion above has rested on that no two corners are met at the same time, but nothing appears to indicate a different conclusion, if this were not so, and the examination of such a coincidental circumstance is left for the future.

\subsection{Average cost approach}

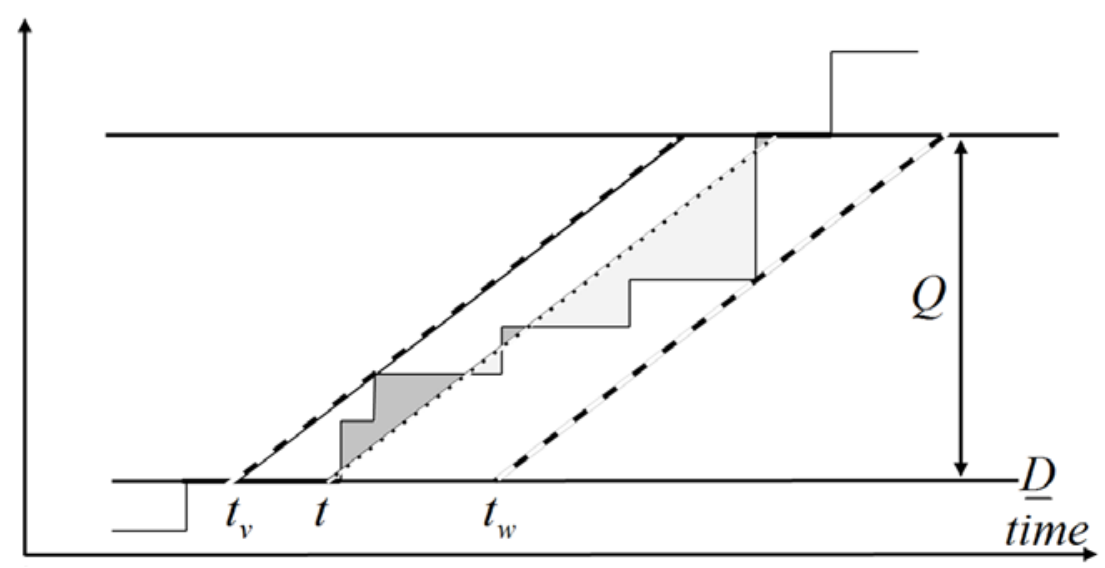

Figure 9. Time-weighted inventory represented by light-shaded areas and time-weighted backlogs by dark shaded areas.

When applying the less sophisticated AC approach instead of using the NPV as the objective function, the timing of setups is disconsidered, which makes the optimisation of timing a batch a question of minimising the sum of inventory holding costs and backlogging costs.

Figure 9 illustrates consequences from moving the production ramp from the left to the right. To the left of $t_{v}$, there are no backlogs (the sets $\mathrm{S}_{2}$ and $\mathrm{S}_{3}$ are empty). In between $t_{v}$ and $t_{w}$ there are both inventories and backlogs, and beyond $t_{w}$ only backlogs (all steps belong to $S_{3}$ ). In this process the time-weighted average of inventories decrease (light shaded areas) and the 
time-weighted area of backlogs (darker shaded areas) increase. Since these time-weighted averages are represented by triangles combined with rectangles, they are easily calculated.

For a step belonging to $\mathrm{S}_{1}$, there is only inventory. Here $t \leq t_{i}-\left(\bar{D}_{i}-\underline{D}\right) / q$ and the contribution to the inventory area will be $D_{i}\left(t_{i}-t+\left(\bar{D}_{i-1}-\underline{D}+D_{i} / 2\right) / q\right)$. For a step belonging to $\mathrm{S}_{2}$ with $t_{i}-\left(\bar{D}_{i}-\underline{D}\right) / q<t \leq t_{i}-\left(\bar{D}_{i-1}-\underline{D}\right) / q$, its inventory area contribution is $\left(t_{i}-t-\left(\bar{D}_{i-1}-\underline{D}\right) / q\right)^{2} q / 2$ and its backlogging area contribution $\left(\left(\bar{D}_{i}-\underline{D}\right) / q-\left(t_{i}-t\right)\right)^{2} q / 2$. Finally, with $i \in \mathrm{S}_{3}$, where $t_{i}-\left(\bar{D}_{i-1}-\underline{D}\right) / q<t$, the contribution to the backlogging area will be $D_{i}\left(t-t_{i}+\left(\bar{D}_{i-1}-\underline{D}+D_{i} / 2\right) / q\right)$. Obviously the inventory area decreases linearly and the backlogging area increases linearly to the left and right of $t_{v}$ and $t_{w}$, respectively, whereas the areas contribute with quadratic terms in time in the intermediate interval.

The sum of time-weighted inventory holding and backlogging costs therefore amounts to

$$
\begin{gathered}
\mathrm{TC}_{\text {holding and backlogging }}=h \sum_{i \in \mathrm{S}_{1}} D_{i}\left(t_{i}-t+\left(\bar{D}_{i-1}-\underline{D}+D_{i} / 2\right) / q\right) \\
+\sum_{i \in \mathrm{S}_{2}}\left(h\left(t_{i}-t-\left(\bar{D}_{i-1}-\underline{D}\right) / q\right)^{2}+b\left(\left(\bar{D}_{i}-\underline{D}\right) / q+t-t_{i}\right)^{2}\right) q / 2 \\
+b \sum_{i \in \mathrm{S}_{3}} D_{i}\left(t-t_{i}+\left(\bar{D}_{i-1}-\underline{D}+D_{i} / 2\right) / q\right),
\end{gathered}
$$

and its derivatives are

$$
\frac{d \mathrm{TC}_{\text {holding and backlogging }}}{d t}=-h \sum_{i \in \mathrm{S}_{1}} D_{i}+\sum_{i \in \mathrm{S}_{2}}(h+b)\left(q\left(t-t_{i}\right)+\bar{D}_{i-1}-\underline{D}\right)+b \sum_{i \in \mathrm{S}_{2} \cup S_{3}} D_{i},
$$

and

$$
\frac{d^{2} \mathrm{TC}_{\text {holding and backlogging }}}{d t^{2}}=\sum_{i \in \mathrm{S}_{2}}(h+b) q=(h+b) q n_{\mathrm{S}_{2}} \geq 0,
$$

where $n_{\mathrm{S}_{2}}$ is the number of elements in $\mathrm{S}_{2}$. An interior extreme point is therefore a cost minimum.

It is easily seen that the first derivative of $\mathrm{TC}_{\text {holding and backlogging }}$ is continuous and that the second derivative makes jumps as the ramp passes a new step. At an upper corner at $t_{k}$, where $t=t_{k}-\left(\bar{D}_{k}-\underline{D}\right) / q$, we have an index $k$ moving from $\mathrm{S}_{1}$ to $\mathrm{S}_{2}$ and the difference in $d \mathrm{TC}_{\text {holding and backlogging }} / d t$ becomes zero (using $\bar{D}_{k}=\bar{D}_{k-1}+D_{k}$ ). Similarly, when passing a lower corner at $t_{m}$, where $t=t_{m}-\left(\bar{D}_{m-1}-\underline{D}\right) / q$, the index $m$ moves from $\mathrm{S}_{2}$ to $\mathrm{S}_{3}$ and the difference in the derivative again is zero. Since the second derivative is proportional to the number of elements in $S_{2}$, it makes a jump upwards as a new upper corner enters, and downwards as the lower corner is left. 
In order to compare this AC approach with using the NPV as objective, we expand the derivative of $\mathrm{NPV}_{\text {batch }}(t)$ in (25) into a first-order Maclaurin series, disregarding the time dependence of the setup costs (as is standard in the AC approach), we have

$$
\begin{gathered}
{\left[\frac{d \mathrm{NPV}_{\text {batch }}(t)}{d t}\right]_{1 s t \text { order }}=c q \sum_{i \in \mathrm{S}}\left[\left(1-e^{-\rho D_{i} / q}\right) e^{-\rho\left(t+\left(\bar{D}_{i-1}-\underline{D}\right) / q\right)}\right]_{1 s t \text { order }}+\left[\rho \mathrm{Ke}^{-\rho(t+\beta Q / q)}\right]_{0 \text { th order }}} \\
-p q \sum_{i \in \mathrm{S}_{2}}\left[e^{-\rho t_{i}}\left(1-e^{-\rho\left(t-t_{i}+\left(\bar{D}_{i}-\underline{D}\right) / q\right)}\right)\right]_{1 s t \text { order }}-p q \sum_{i \in \mathrm{S}_{3}}\left[e^{-\rho t}\left(1-e^{-\rho D_{i} / q}\right) e^{-\rho\left(\bar{D}_{i-1}-\underline{D}\right) / q}\right]_{1 s t \text { order }}= \\
=\rho c \sum_{i \in \mathrm{S}} D_{i}-\rho p \sum_{i \in \mathrm{S}_{2}}\left(q\left(t-t_{i}\right)+\bar{D}_{i}-\underline{D}\right)-\rho p \sum_{i \in \mathrm{S}_{3}} D_{i},
\end{gathered}
$$

since $\left[\rho K e^{-\rho(t+(1-\beta) Q / q)}\right]_{0 t h \text { order }}=0$. Rearranging the terms and interpreting $h$ as $\rho c$ and $b$ as $\rho(p-c)$, we obtain

$$
\begin{gathered}
{\left[\frac{d \mathrm{NPV}_{\text {batch }}(t)}{d t}\right]_{1 \text { st order }}=\rho c \sum_{i \in \mathrm{S}_{1}} D_{i}-\sum_{i \in \mathrm{S}_{2}} \rho p\left(q\left(t-t_{i}\right)+\bar{D}_{i-1}-\underline{D}\right)-\rho(p-c) \sum_{i \in \mathrm{S}_{2} \cup \mathrm{S}_{3}} D_{i}=} \\
=h \sum_{i \in \mathrm{S}_{1}} D_{i}-\sum_{i \in \mathrm{S}_{2}}(h+b)\left(q\left(t-t_{i}\right)+\bar{D}_{i-1}-\underline{D}\right)-b \sum_{i \in \mathrm{S}_{2} \cup \mathrm{S}_{3}} D_{i},
\end{gathered}
$$

which coincides perfectly with $-d \mathrm{TC}_{\text {holding and backlogging }} / d t$ in (25). This again shows the $\mathrm{AC}$ approach to be a first/zeroth-order approximation of adopting the NPV principle.

\subsection{Optimising the production structure}

The question of choosing an optimal production structure (an optimal sequence of consecutive production ramps) is to choose individually optimised batches together covering all demand steps such that an overall objective function is maximised. Figure 10 illustrates how batches are matched together. In this figure each intersection between the dashed lines represents the end and start levels of two adjacent batches. Each arrow, horizontal or vertical, represents a batch. A lower corner $\left(\bar{D}_{i}, \bar{D}_{j}\right)$ (between a horizontal arrow and a following vertical arrow) indicates that an old batch started at level $\bar{D}_{j}$ and ended at level $\bar{D}_{i}$, from which the new batch starts. An upper corner $\left(\bar{D}_{i}, \bar{D}_{k}\right)$ (between a vertical arrow and a following horizontal arrow) shows the old batch to have started at level $\bar{D}_{i}$ and ended at level $\bar{D}_{k}$, from which the new batch begins.

A production structure is given by a sequence of adjoining arrows, always beginning at $\bar{D}_{0}=0$ and ending at $\bar{D}_{n}$, each crossing between the shaded and the unshaded areas in Figure 10. There are $2^{n-1}$ possible paths. In the right part of the figure are illustrated the LotFor-Lot (L4L) and the “All-At-Once” ( $\forall @ 1$ ) structures. 

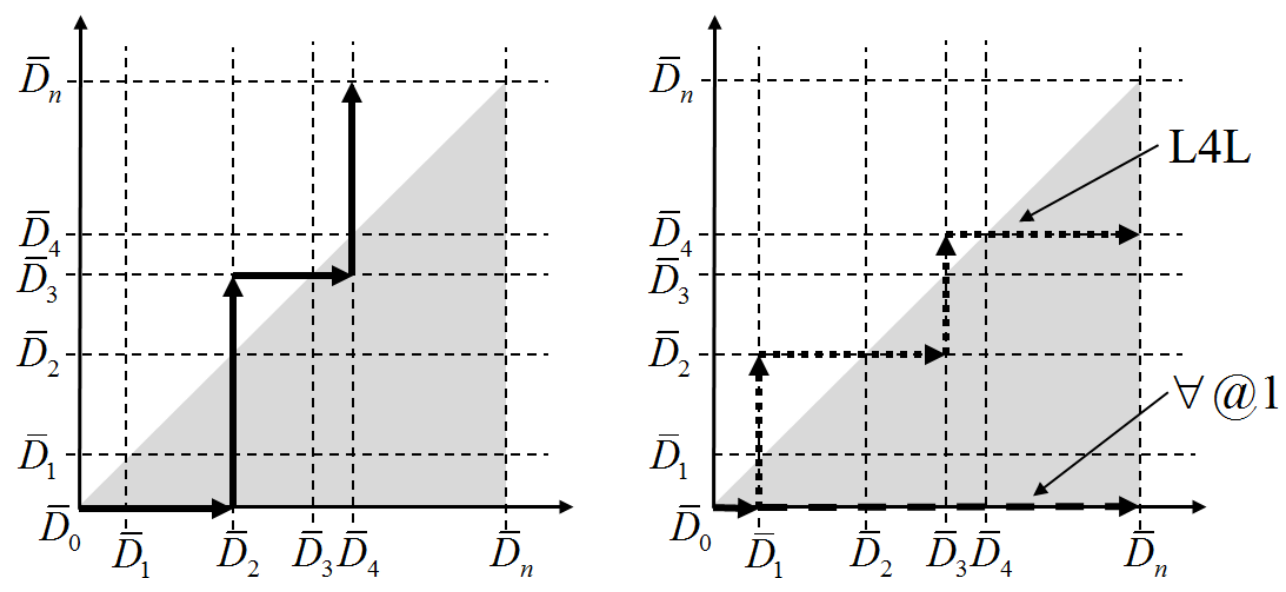

Figure 10. A production structure represented by a path between cumulative demand levels. The right-hand part of the figure illustrates the two extreme Lot-For-Lot and All-At-Once structures.

A question that has not been addressed is whether the individual solutions to optimising batches might provide start times in conflict with each other for adjacent ramps. Would it be possible that an optimal batch on one level required a start time that was earlier than the ending time of an earlier batch (i.e. a batch covering earlier steps of the demand staircase), and, if so, under which circumstances?

This situation with an optimal anterior ramp followed by an optimal posterior ramp starting earlier than the ending time of the anterior ramp is possible since the ramps are individually optimised. The likelihood of this possibility increases, if the anterior ramp holds steep steps and the posterior ramp flat steps. A necessary condition for such a possibility is $t_{v}^{\prime \prime}<t_{w}^{\prime}+Q^{\prime} / q$, where reference is made to Figure 8 and where primes refer to the anterior batch and the double-prime to the posterior batch.

One might consider handling this situation by introducing the restriction $t^{\prime}+Q^{\prime} / q \leq t^{\prime \prime}$ (with the two timing decision variables $\left(t^{\prime}, t^{\prime \prime}\right)$ to the maximisation problem adding the two NPV functions from Eq. (4) together and including a non-negative multiplicator $\lambda$ in a Lagrangean function $\mathcal{L}=\mathrm{NPV}_{\text {batch }}\left(t^{\prime}\right)+\mathrm{NPV}_{\text {batch }}\left(t^{\prime \prime}\right)+\lambda\left(t^{\prime \prime}-t^{\prime}-Q^{\prime} / q\right)$. Due to the unimodal properties of the two functions $\operatorname{NPV}_{\text {batch }}\left(t^{\prime}\right)$ and $\operatorname{NPV}_{\text {batch }}\left(t^{\prime \prime}\right)$, the joint optimisation will lead to a positive $\lambda$ requiring an equality $t^{\prime \prime}=t^{\prime}+Q^{\prime} / q$, if the individually optimised solutions happen to obey $t^{\prime \prime}<t^{\prime}+Q^{\prime} / q$. This means that the two ramps merge and also that one of the setup costs may be saved.

However, the solution with two adjacent ramps made into one alone, is already covered by one of the other production structures treated in the space of solutions. So the only measure to be taken when a timing conflict of this kind appears when matching batches into a structure, is to exclude this particular structure from further consideration.

\section{Numerical example}

We choose to apply our developments to the numerical example of Roger M. Hill (Hill 1997), also applied in (Grubbström 2012). Since the investigations in these two pieces of work concerned only costs/out-payment consequences, we now need to extend our parameters also with a unit sales price value. 
The requirements and their timing are given in Table 2.

\begin{tabular}{|l|c|c|c|c|c|c|c|c|c|c|}
\hline Event no $j$ & 1 & 2 & 3 & 4 & 5 & 6 & 7 & 8 & 9 & 10 \\
\hline Time $_{j}$ & 3 & 4 & 6 & 8 & 9 & 10 & 14 & 15 & 19 & 20 \\
\hline${\text { Amount required } D_{j}}$ & 8 & 6 & 8 & 4 & 6 & 7 & 8 & 5 & 9 & 7 \\
\hline
\end{tabular}

Table 2. Requirements and their timing in example.

The same parameter values as in (Hill 1997) are assumed, namely a setup cost $K=36$ and a production rate $q=5$. The unit production cost is set to $c=10$, unit sales price to $p=15$, and the continuous interest rate to $\rho=0.1$, making the holding costs parameter $h=1$, when using the interpretation $h=\rho c$. The setup cost is assumed to be located at the beginning of a production ramp $\beta=0$.

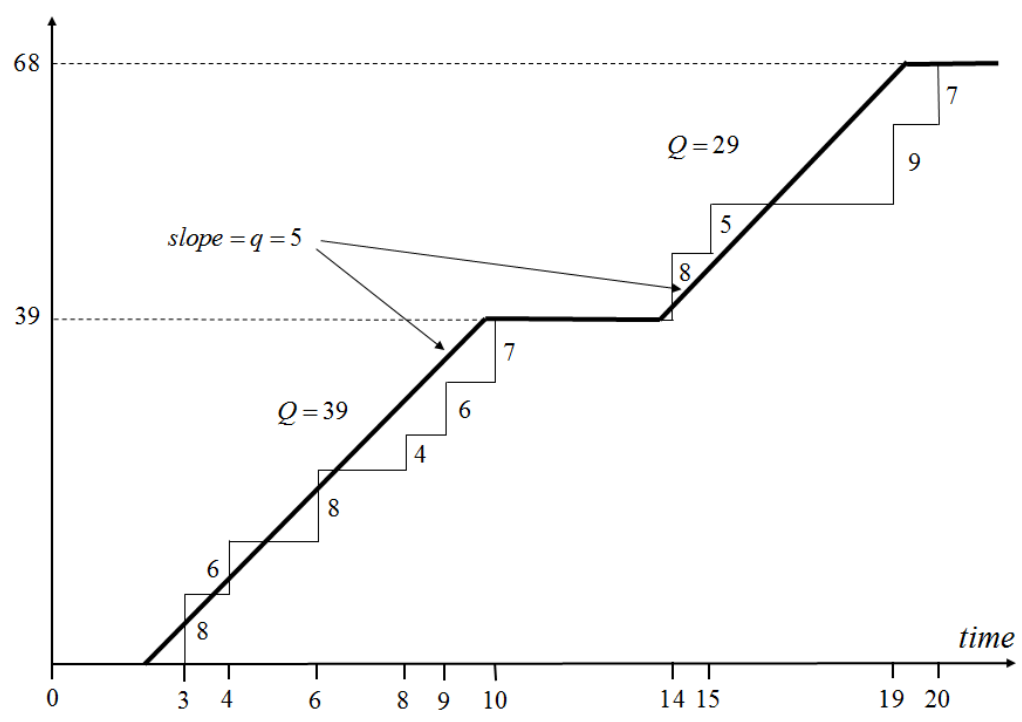

Figure 11. The demand staircase of the example in Hill (1997) together with a twobatch production structure.

In Figure 11 the cumulative demand staircase is shown with thinly outlined steps. An example of cumulative production with two batches is also shown with two thick ramps having the slope $q=5$. With this production, events 1,2 , and 3 belong to the set $\mathrm{S}_{2}$ of the first batch, and events 4,5 and 6 to the set $S_{1}$. For the second batch, the event 7 belongs to $S_{2}$, event 8 to $S_{1}$, and events 9 and 10 to $S_{3}$. The number of structures eligible for optimality is $2^{10-1}=512$.

After examining all structures, and taking a local optimisation of the batches belonging to these structures, the two-batch structure shown in Figure 11 is found to be optimal. The first batch covers the first six demand events, and the second batch the remaining four events.

Letting the start time of these batches vary, the NPV (solid curve) of the batch, its first derivative (dashed curve) and second derivative (dotted curve) behaved as shown in Figure 12 (first batch) and Figure 13 (second batch). The vertical scale for the three curves has been 
chosen so as to make $\rho^{2} \mathrm{NPV}_{\text {batch }}, \rho d \mathrm{NPV}_{\text {batch }} / d t$ and $d^{2} \mathrm{NPV}_{\text {batch }} / d t^{2}$ comparable. The properties in (22) and (23) are clearly verified.

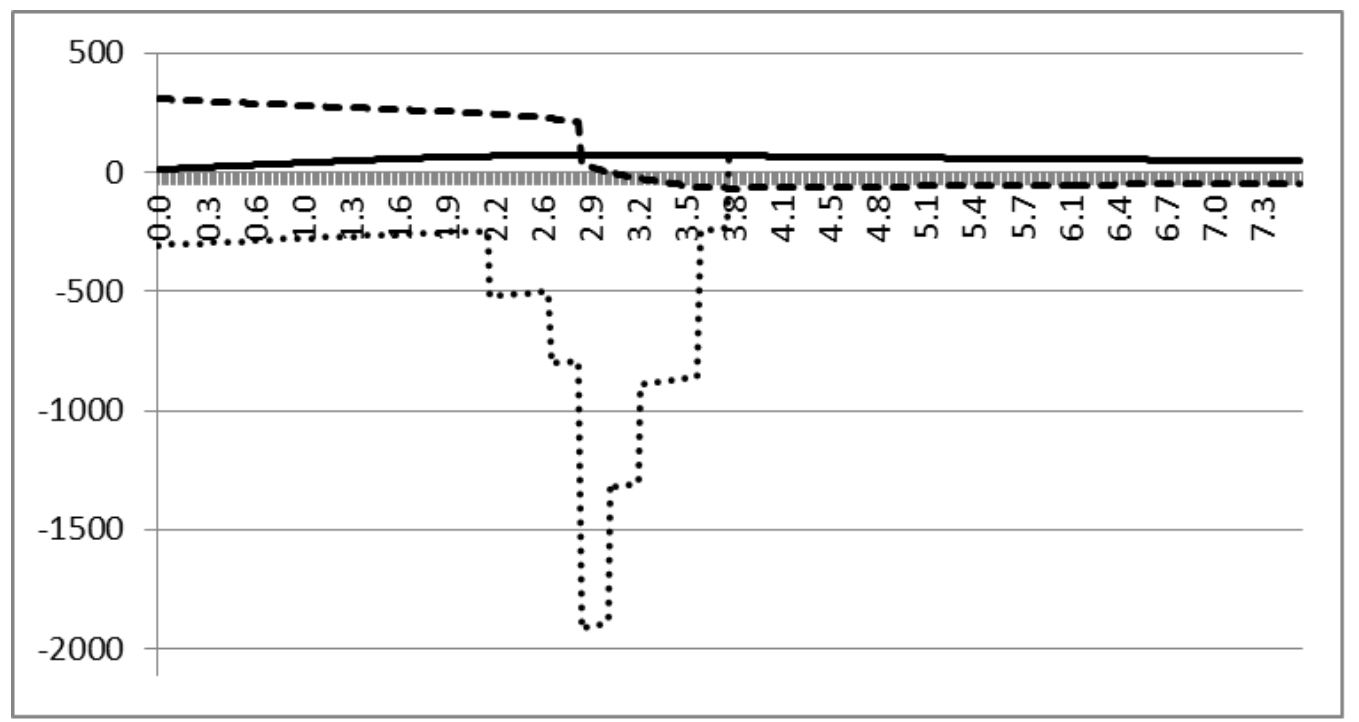

Figure 12. NPV of first optimal batch and its derivatives as a function of start time. Solid curve shows NPV, dashed curve first derivative, and dotted curve second derivative.

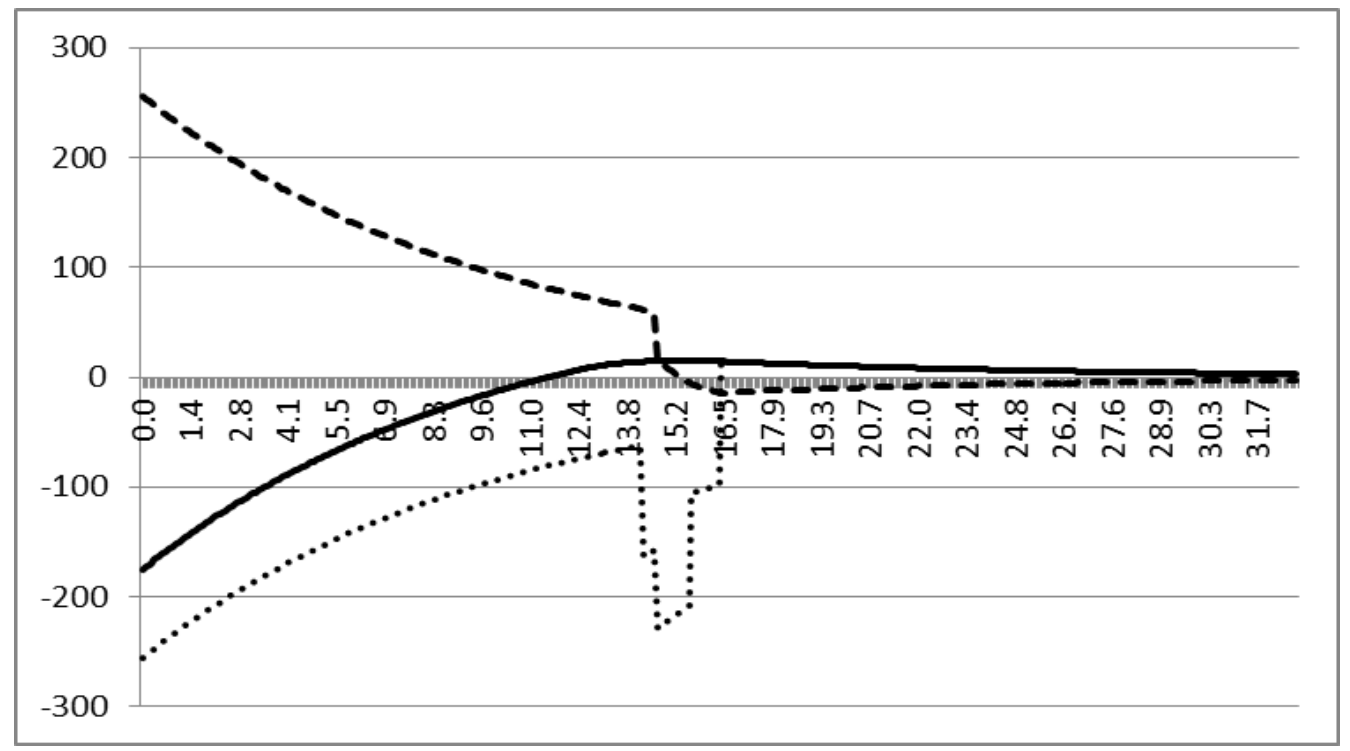

Figure 13. NPV of second optimal batch and its derivatives as a function of start time. Solid curve shows NPV, dashed curve first derivative, and dotted curve second derivative.

All $n(n+1) / 2=55$ individually optimised batch solutions are listed in Table 3. Here, columns represent the first demand step covered by a batch and rows the last demand step covered. At the top of a column is marked the prior cumulative demand level $\bar{D}_{i-1}$ of a demand event at $t_{i}$, and to the left in a row the posterior cumulative demand level $\bar{D}_{j}$ from a (later) event at $t_{j}$. The batch size is the difference between $\bar{D}_{j}$ and $\bar{D}_{i-1}$. 


\begin{tabular}{|c|c|c|c|c|c|c|c|c|c|c|c|c|}
\hline \multicolumn{3}{|r|}{$\bar{D}_{i-1}$} & $\mathbf{0}$ & 8 & 14 & 22 & 26 & 32 & 39 & 47 & 52 & 61 \\
\hline $\bar{D}_{j}$ & $t_{j}$ & $t_{i}$ & 3 & 4 & 6 & 8 & 9 & 10 & 14 & 15 & 19 & 20 \\
\hline \multirow{2}{*}{8} & \multirow{2}{*}{3} & $t$ & 2.99 & & & & & & & & & \\
\hline & & NPV & 0.71 & & & & & & & & & \\
\hline \multirow{2}{*}{14} & \multirow{2}{*}{4} & $t$ & 2.40 & 4 & & & & & & & & \\
\hline & & NPV & 18.60 & $\begin{array}{l}-5.18 \\
\end{array}$ & & & & & & & & \\
\hline \multirow{2}{*}{22} & \multirow{2}{*}{6} & $t$ & 2.67 & 4.42 & 5.99 & & & & & & & \\
\hline & & NPV & 39.68 & 15.80 & 0.53 & & & & & & & \\
\hline \multirow{2}{*}{26} & \multirow{2}{*}{8} & $t$ & 2.85 & 4.66 & 6.12 & 8 & & & & & & \\
\hline & & NPV & 47.87 & 24.30 & 9.27 & -7.54 & & & & & & \\
\hline \multirow{2}{*}{32} & \multirow{2}{*}{9} & $t$ & 2.961 & 4.76 & 6.17 & 8.06 & 9.00 & & & & & \\
\hline & & NPV & 59.19 & 35.87 & 20.98 & 4.13 & -3.14 & & & & & \\
\hline \multirow{2}{*}{39} & \multirow{2}{*}{10} & $t$ & 2.99 & 4.76 & 6.10 & 7.89 & 8.75 & 10.00 & & & & \\
\hline & & NPV & 71.43 & 48.16 & 33.22 & 16.20 & 8.75 & -1.23 & & & & \\
\hline \multirow{2}{*}{47} & \multirow{2}{*}{14} & $t$ & 4.60 & 6.20 & 7.40 & 9.36 & 10.50 & 12.15 & 13.99 & & & \\
\hline & & NPV & 70.95 & 50.97 & 37.95 & 22.98 & 16.63 & 8.35 & 0.24 & & & \\
\hline \multirow{2}{*}{52} & \multirow{2}{*}{15} & $t$ & 4.60 & 6.20 & 7.50 & 9.51 & 10.49 & 11.93 & 13.40 & 15.00 & & \\
\hline & & NPV & 75.95 & 55.97 & 42.97 & 28.33 & 22.03 & 13.65 & 5.22 & -2.72 & & \\
\hline \multirow{2}{*}{61} & \multirow{2}{*}{19} & $t$ & 6.80 & 8.40 & 9.60 & 11.20 & 12.00 & 13.41 & 15.44 & 17.62 & 18.93 & \\
\hline & & NPV & 69.09 & 53.06 & 42.62 & 30.50 & 25.13 & 17.87 & 10.78 & 4.22 & 0.78 & \\
\hline \multirow{2}{*}{68} & \multirow[b]{2}{*}{20} & $t$ & 6.80 & 8.40 & 9.60 & 11.20 & 12.04 & 13.57 & 15.30 & 17.20 & 18.20 & 20.00 \\
\hline & & NPV & 73.45 & 57.42 & 46.98 & 34.86 & 29.49 & 22.35 & 15.28 & 8.54 & 4.89 & -0.45 \\
\hline
\end{tabular}

Table 3. Individually optimised batches of example, each with optimal start time t (above), and $\mathrm{NPV}_{\text {batch }}$ (below. The light-shaded pair of cells shows a batch of size 47-14=33 starting

on level $\bar{D}_{i-1}=14$ with an optimal start time of 7.40 and a resulting NPV contribution of

37.95. The two batches belonging to the optimal structure are indicated by bold frames.

The optimal solution is also reported in Table 4. The solution to the currently treated problem, in which shortages are allowed, is compared to the solution to the similar problem if shortages were not allowed.

\begin{tabular}{|l|c|c|c|c|c|c|c|}
\hline & \multicolumn{2}{|c|}{ Start time $t$} & \multirow{2}{*}{\begin{tabular}{c} 
No \\
\cline { 5 - 7 }
\end{tabular}} & Optimal & & \multicolumn{4}{|c|}{ NPV } \\
\hline Optimal & $\begin{array}{c}\text { Nortages } \\
\text { sholay } \\
\text { allowed }\end{array}$ & & Maximum & $\begin{array}{c}\text { No } \\
\text { shortages } \\
\text { allowed }\end{array}$ & $\begin{array}{c}\text { Improvement } \\
\text { in NPV }\end{array}$ \\
\hline Batch 1 & 2.99 & 1.2 & $\mathbf{1 . 8}$ & 71.43 & 45.16 & $\mathbf{2 6 . 2 7}$ & $\mathbf{5 8 \%}$ \\
\hline Batch 2 & 15.30 & 12.4 & $\mathbf{2 . 9}$ & 15.28 & 6.63 & $\mathbf{8 . 6 5}$ & $\mathbf{1 3 0 \%}$ \\
\hline Total & & & & $\mathbf{8 6 . 7 0}$ & $\mathbf{5 1 . 7 9}$ & $\mathbf{3 4 . 9}$ & $\mathbf{6 7 \%}$ \\
\hline
\end{tabular}

Table 4. Optimal solution compared to solution of problem when assuming no shortages allowed.

Figures 14 and 15 include scatter diagrams of all 512 structures showing their NPV against number of batches for each structure after optimising the timing of each batch. It is seen in Figure 14 that the optimal solution is found for a structure with two batches, and that the worst case solution (lowest NPV) happens to be obtained for the Lot-For-Lot solution (10 batches with an NPV of -18.0). Figure 15 shows the same type of diagram for the problem, when no shortages are allowed, and Figure 16 the improvement in NPV for these solutions, when the no shortage restriction is relaxed. 


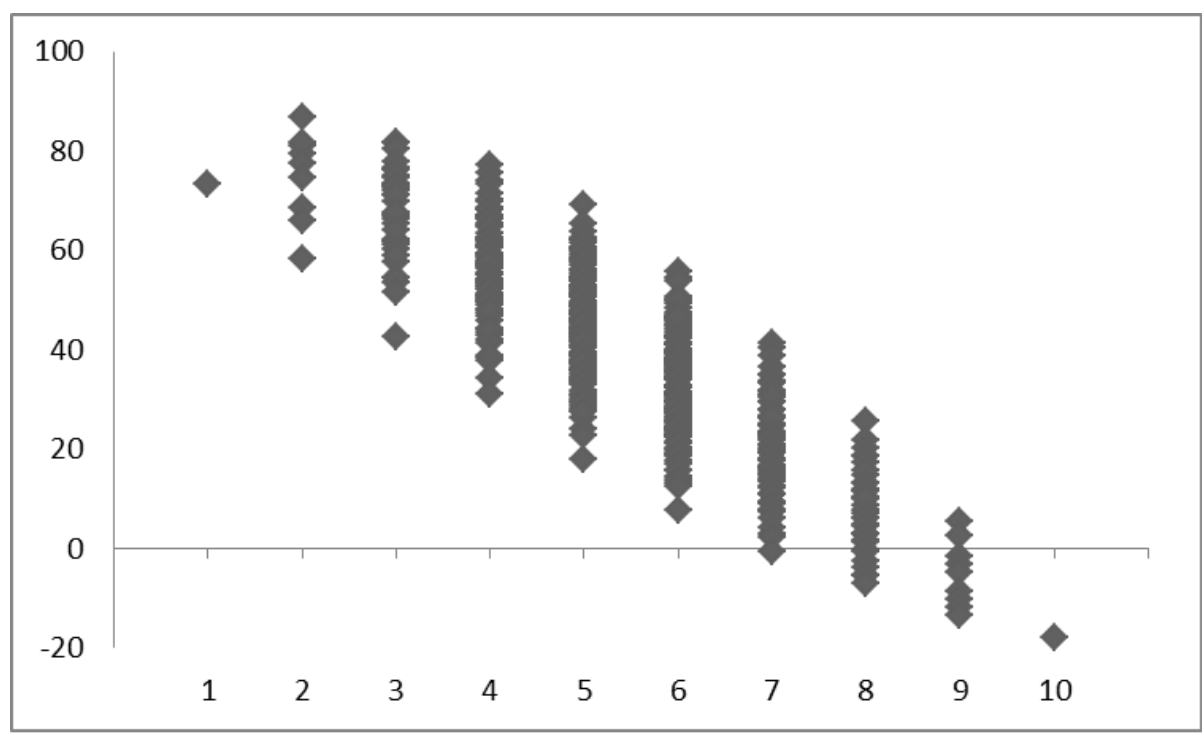

Figure 14. Scatter diagram of NPV against number of batches for all possible structures, when backlogs are allowed.

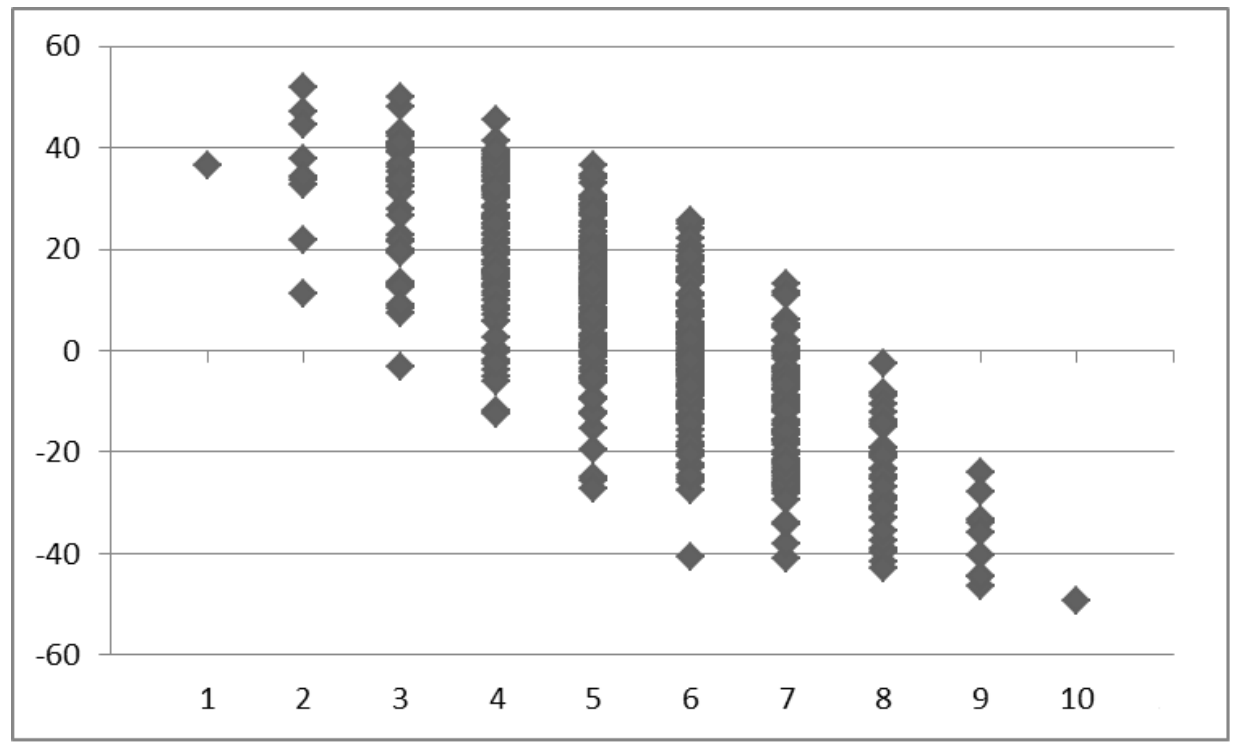

Figure 15. Scatter diagram of NPV against number of batches for all possible structures, when backlogs are not allowed. 


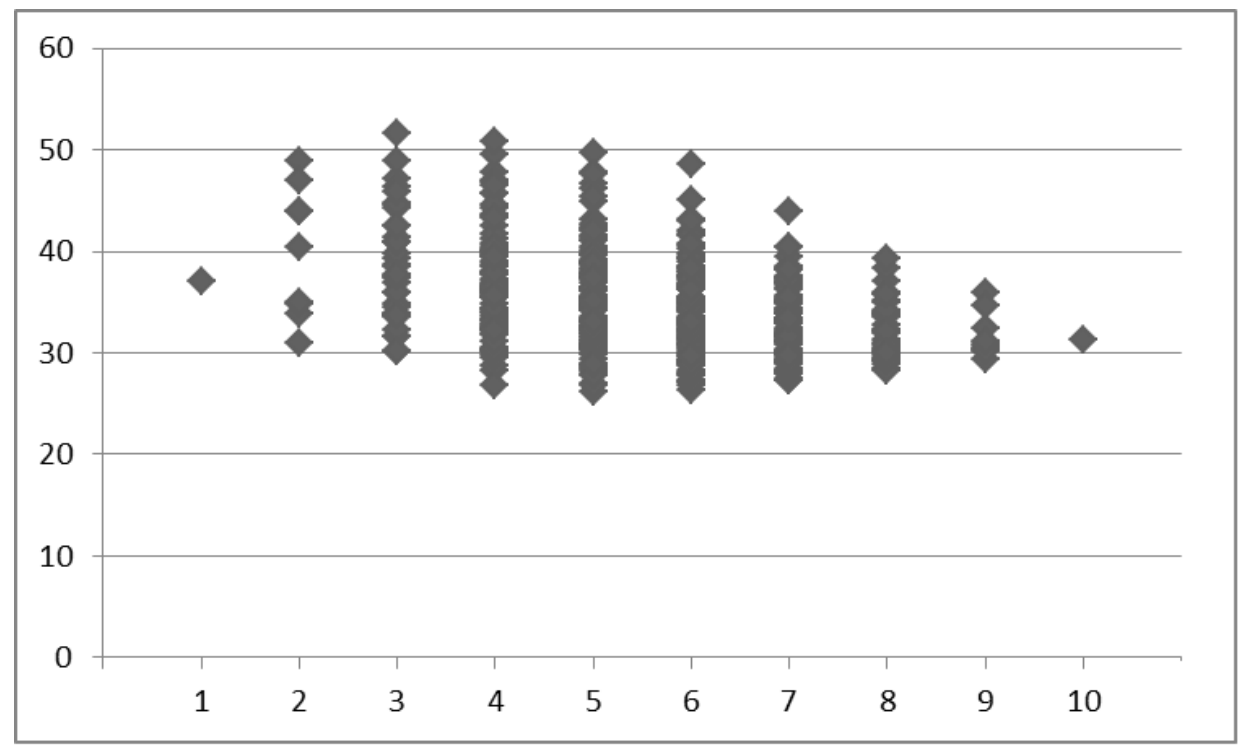

Figure 16. Scatter diagram showing improvement in NPV for each structure against number of batches, when allowing shortages.

In Figure 17 is displayed the resulting NPV when applying the AC approach and minimising total cost, again against number of batches. Figure 18 shows the loss in NPV by using the AC approach.

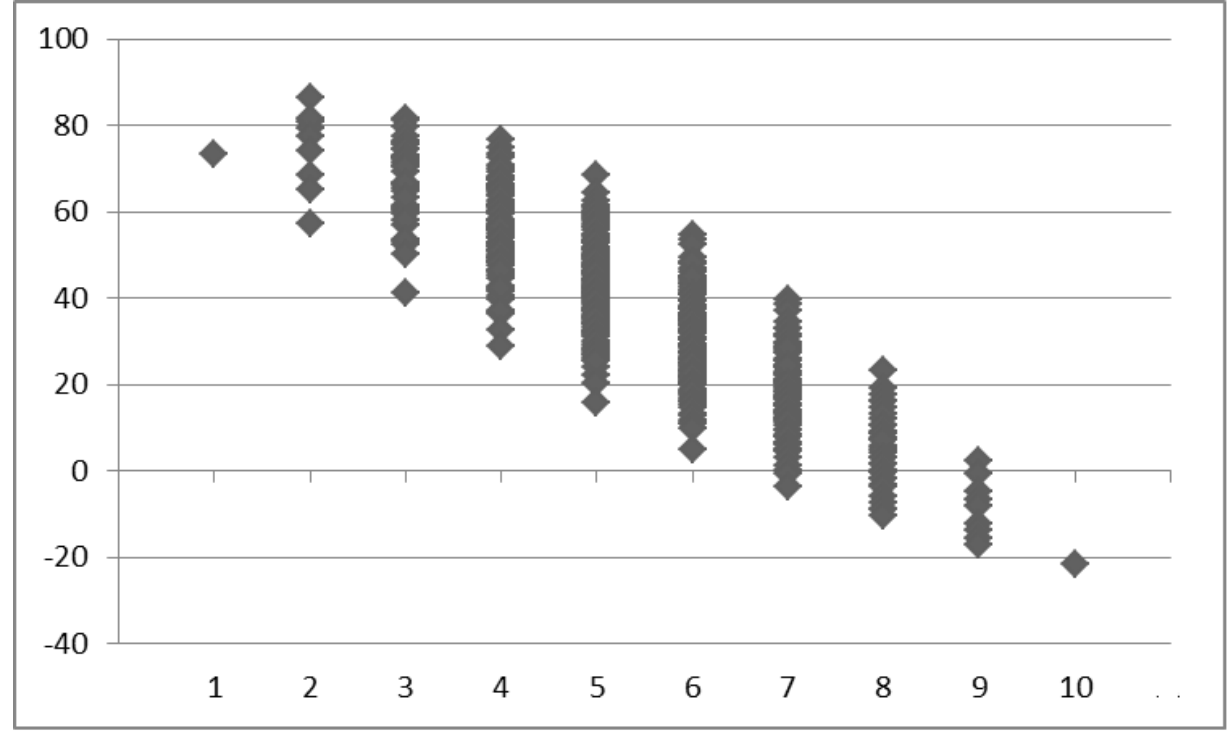

Figure 17. Values of NPV when minimising total cost, against number of batches. 


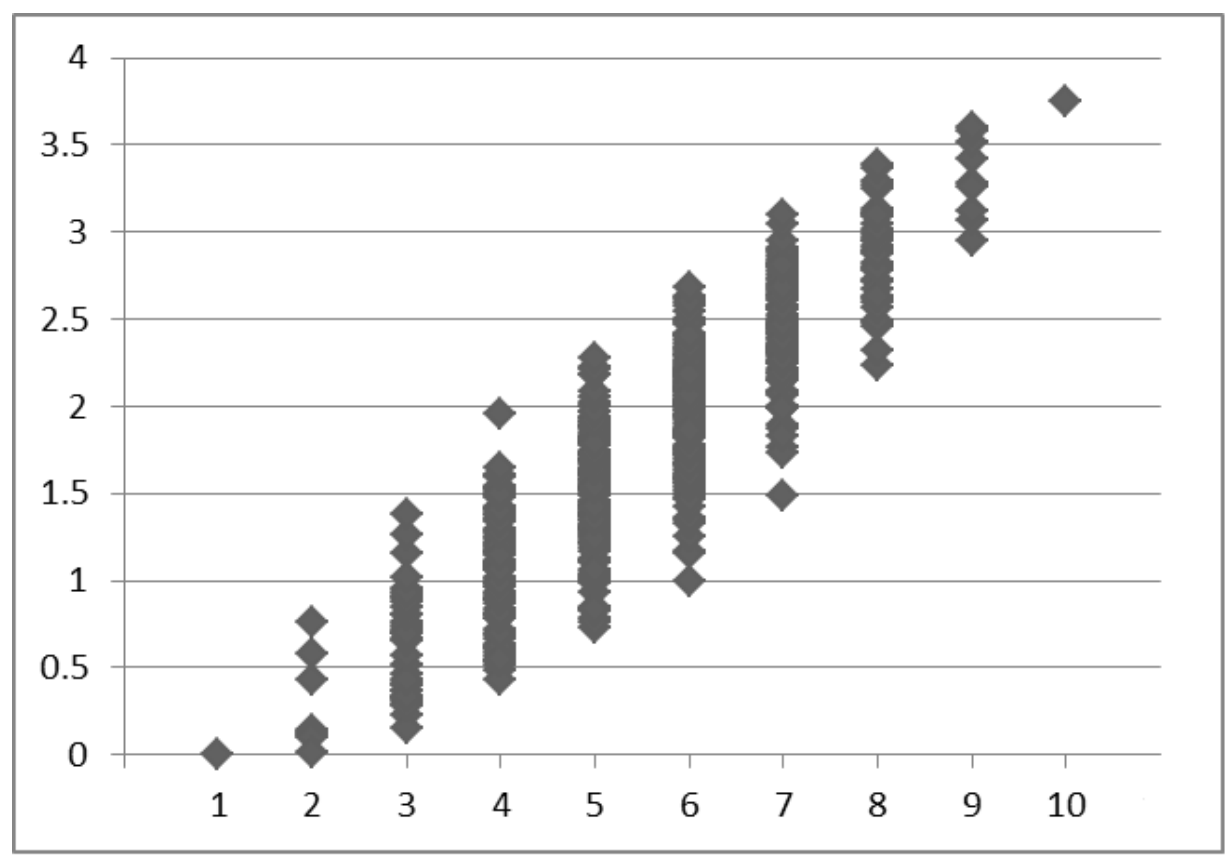

Figure 18. Loss in NPV from applying AC approach instead of maximising NPV, against number of batches.

\section{Conclusions}

In the foregoing we have examined consequences of relaxing the no-shortage assumption in dynamic lotsizing. Assuming a finite production rate, it was first shown that the segments (ramps) of the optimal cumulative production function must start and end at levels given by the horizontal steps of cumulative demand. This limits the number of structures eligible for optimality to the finite number $2^{n-1}$, but the batches belonging to each structure need to be optimised as to their start times separately.

Each segment in the production structure (batch) can be optimised individually providing a set of $n(n+1) / 2$ batches to choose from, when selecting the optimal production structure.

It was also shown that with shortages allowed, the optimal production ramps necessarily intersect the demand staircase. This means that some shortage is always optimal.

A numerical example was included illustrating a case with ten demand events and therefore $2^{9}=512$ possible structures with 55 batch sizes included in the set of structures. The optimum structure was formed by two batches in this case.

Items for further studies would be to include the opportunity of fixed shortage costs to represent, for instance, badwill, and to extend the analysis to combining backlogs and lost sales opportunities.

\section{References}

Beullens, P., Janssens, G. K., 2011. Holding costs under push or pull conditions - The impact of the Anchor Point, European Journal of Operational Research, 215, 115-125.

Grubbström, R. W., 1980. A principle for determining the correct capital costs of work-inprogress and inventory, International Journal of Production Research, 18, 259-271. 
Grubbström, R. W., 2012. Dynamic Lotsizing with a Finite Production Rate, International Journal of Production Economics, Article in Press, doi: 10.1016/j.ijpe.2012.12.009.

Grubbström, R. W., Bogataj, M., Bogataj, L., 2009. Optimal Lotsizing within MRP Theory, Annual Reviews in Control, 34(1), 2010, 89-100.

Grubbström, R. W., Tang, O., 2012. The space of solution alternatives in the optimal lotsizing problem for general assembly systems applying MRP theory, International Journal of Production Economics, 140(2), 765-777.

Hadley, G., 1964. A comparison of order quantities using the average annual costs and the discounted costs, Management Science, 10, 472-476.

Harris, F. W., 1913. "How Many Parts to Make at Once," Factory, The Magazine of Management, 10(2), 135-136, 152.

Hill, R. M., 1997. Note: Dynamic Lot Sizing for a Finite Rate Input Process, Naval Logistics Research, 44(2), 221-228.

Kim, Y. H., Philippatos, G. C., Chung, K. H., 1986. Evaluating Investment in Inventory Policy: A Net Present Value Framework, The Engineering Economist, 31, 119-136.

Song, Y., Chan, G.H., (2005). Single item lot-sizing problems with backlogging on a single machine at a finite production rate, European Journal of Operational Research, 161(1), 191-202.

Teunter, R., van der Laan, E., 2002. On the non-optimality of the average cost approach for inventory models with remanufacturing, International Journal of Production Economics, 79(1), 67-73.

Trippi, R. R., Levin, D. E., 1974. A present value formulation of the classical EOQ problem, Decision Sciences, 5, 30-35.

Veinott Jr., A. F., 1969. Minimum concave-cost solution of Leontief substitution models of multifacility inventory systems. Operations Research, 17, 262-291.

Wagner, H. M., Whitin, T. M., 1958. A Dynamic Version of the Economic Lot Size Model, Management Science, 5, 89-96. 